\title{
CLIC1 recruits PIP5K1A/C to induce cell-matrix adhesions for tumor metastasis
}

\author{
Jei-Ming Peng, ${ }^{1,2}$ Sheng-Hsuan Lin, ${ }^{1}$ Ming-Chin Yu, ${ }^{3}$ and Sen-Yung Hsieh ${ }^{1,4}$ \\ 'Department of Gastroenterology and Hepatology, Chang Gung Memorial Hospital, Linkou, Taoyuan, Taiwan. ${ }^{2}$ Institute for Translational Research in Biomedicine, Kaohsiung Chang Gung Memorial Hospital, \\ Kaohsiung, Taiwan. ${ }^{3}$ Department of General Surgery, Chang Gung Memorial Hospital, Linkou, Taoyuan, Taiwan. ${ }^{4}$ Chang Gung University College of Medicine, Taoyuan, Taiwan.
}

\begin{abstract}
Membrane protrusion and adhesion to the extracellular matrix, which involves the extension of actin filaments and formation of adhesion complexes, are the fundamental processes for cell migration, tumor invasion, and metastasis. How cancer cells efficiently coordinate these processes remains unclear. Here, we showed that membrane-targeted chloride intracellular channel 1 (CLIC1) spatiotemporally regulates the formation of cell-matrix adhesions and membrane protrusions through the recruitment of PIP5Ks to the plasma membrane. Comparative proteomics identified CLIC1 upregulated in human hepatocellular carcinoma (HCC) and associated with tumor invasiveness, metastasis, and poor prognosis. In response to migration-related stimuli, CLIC1 recruited PIP5K1A and PIP5K1C from the cytoplasm to the leading edge of the plasma membrane, where PIP5Ks generate a phosphatidylinositol 4,5-bisphosphate-rich (PIP ${ }_{2}$-rich) microdomain to induce the formation of integrin-mediated cell-matrix adhesions and the signaling for cytoskeleon extension. CLIC1 silencing inhibited the attachment of tumor cells to culture plates and the adherence and extravasation in the lung alveoli, resulting in suppressed lung metastasis in mice. This study reveals what we believe is an unrecognized mechanism that spatiotemporally coordinates the formation of both lamellipodium/invadopodia and nascent cell-matrix adhesions for directional migration and tumor invasion/metastasis. The unique traits of upregulation and membrane targeting of CLIC1 in cancer cells make it an excellent therapeutic target for tumor metastasis.
\end{abstract}

\section{Introduction}

Membrane protrusion and adhesion to the extracellular matrix are the 2 fundamental processes of different modes of cell migration, which are crucial for embryonic development, wound healing, immune responses, and tumor invasion and metastasis (1, 2). Membrane protrusion, a de novo membrane extension in the direction of movement forming the leading edge as filopodia, lamellipodia, or invadopodia, results from the expansion of the cytoskeleton (actin filaments or F-actin, which serves as a scaffold for myosin II motors and other effectors). Adhesion to the extracellular matrix occurs through the formation of adhesion complexes, namely cell-matrix adhesions. Cell-matrix adhesions are membranous hubs that mediate the interaction between the extracellular matrix and the cellular scaffolding and signaling machinery via membranous receptors, mainly integrins, for extracellular ligands. Integrins, a large superfamily of heterodimeric receptors, bind to diverse extracellular matrix molecules to trigger intracellular signals $(3,4)$. Talin is required for most integrin-mediated adhesive functions $(5,6)$ and is thus viewed as a central player in integrin functions, such as adhesion for migration $(7,8)$. Binding of talin to the cytoplasmic domain of integrin $\beta$ leads to the formation

Authorship note: JMP and SHL contributed equally to this work.

Conflict of interest: The authors have declared that no conflict of interest exists. Copyright: () 2021, American Society for Clinical Investigation.

Submitted: October 7, 2019; Accepted: October 14, 2020; Published: January 4, 2021

Reference information: / Clin Invest. 2021;131(1):e133525.

https://doi.org/10.1172/JCl133525. of integrin $\alpha \beta$ heterodimers, resulting in the assembly of nascent cell-matrix adhesions, which then elicit adhesion-mediated signals regulating actin cytoskeleton polymerization and cell motility (9). Therefore, the formation of nascent cell-matrix adhesions plays a pivotal role in cell adherence to the matrix and signaling for extension of the actin cytoskeleton during cell migration (10). Interestingly, accumulating evidence suggests the involvement of deregulated cell-matrix adhesions in cancer development and tumor metastasis $(11,12)$. However, how cells, particularly tumor cells with high invasiveness, spatiotemporally regulate cell-matrix adhesion and the formation of filopodia, lamellipodia, and invadopodia for directional migration remains unclear.

The chloride intracellular channel (CLIC) family consists of 6 highly conserved members (CLIC1-6). All CLICs consist of an $\mathrm{N}$-terminal thioredoxin-like domain followed by an $\alpha$-helix C-terminal domain (13). The CLICs are unique among eukaryotic ion channels in that they can exist as either a soluble monomer or an integral membrane channel upon undergoing structural rearrangement (14). These proteins play diverse physiological roles in health and diseases, including inflammatory responses to tissue injury and tumor progression (15). CLIC1 is the most studied because of its broad expression in different mammalian tissues and cells (16). In response to stimuli, such as oxidative stress, CLIC1 changes its structure and is inserted into lipid membranes to form an ion channel in vitro $(14,17,18)$. In macrophages, CLIC1 inserts into the phagosomal membrane upon phagocytosis. Increasing evidence has shown that CLIC1 can insert into the plasma membrane (19) and function as a chloride ion channel (20), although these findings are 
Table 1. Correlation of CLIC1 levels to clinical manifestations in 89 patients with HCC

\begin{tabular}{|c|c|c|c|c|c|c|}
\hline & Group & Low CLIC level & High CLIC level & Total & $P$ value & Statistical test \\
\hline \multirow[t]{2}{*}{ Sex } & Male & 31 & 31 & 62 & 0.15 & $\chi^{2}$ \\
\hline & Female & 18 & 9 & 27 & & \\
\hline \multirow[t]{6}{*}{ Age, years } & $20-29$ & 1 & 0 & 1 & 0.563 & Kruskal-Wallis \\
\hline & $30-39$ & 1 & 8 & 9 & & \\
\hline & $40-49$ & 21 & 12 & 33 & & \\
\hline & $50-59$ & 17 & 10 & 27 & & \\
\hline & $60-69$ & 8 & 10 & 18 & & \\
\hline & $70-79$ & 1 & 0 & 1 & & \\
\hline \multirow[t]{3}{*}{ Tumor stage } & 1 & 21 & 9 & 30 & 0.006 & Kruskal-Wallis \\
\hline & ॥ & 18 & 12 & 30 & & \\
\hline & IIIB & 10 & 19 & 29 & & \\
\hline \multirow[t]{2}{*}{ Etiology } & HBV & 24 & 21 & 45 & 0.745 & $\chi^{2}$ \\
\hline & $\mathrm{HCV}$ & 25 & 19 & 44 & & \\
\hline \multirow[t]{4}{*}{ Vascular invasion } & 0 & 26 & 13 & 39 & 0.018 & Kruskal-Wallis \\
\hline & 1 & 1 & 1 & 2 & & \\
\hline & 2 & 19 & 18 & 37 & & \\
\hline & $3-4$ & 3 & 8 & 11 & & \\
\hline
\end{tabular}

IHC score: percentage of positive cells $\times$ IHC intensity (0-3); high IHC score $\geq 200$; low IHC score < 200. HBV, hepatitis B virus; HCV, hepatitis C virus. Vascular invasion grade: 0 , no vascular invasion; 1 , capsular vein invasion; 2 , microscopic vascular invasion; 3 , gross vascular invasion; 4 , main branches of the portal or hepatic vein invasion. el: normal liver/early HCC/invasive HCC $=$ 1/4.65/10.09, $P<0.001$; Figure 1, B-D, and Supplemental Table 2). We thus selected CLIC1 for further investigation.

CLIC1 upregulation in HCC is associated with vascular invasion, metastasis, and lower survival. To validate the correlation between CLIC1 upregulation and HCC progression, we used immunohistochemistry (IHC) to compare the CLIC1 levels between the HCC tissues and the paratumor liver tissues on tissue microarrays (TMA) from 89 patients with HCC (Supplemental Figure $1 \mathrm{~A}$ and Supplemental Table 3). The CLIC1 level was significantly increased in the HCC tissues $(P<0.001$ by Student's $t$ and paired $t$ tests; Figure 2A). Moreover, high CLIC1 levels were significantly associated with advanced tumor stages $(P=0.006$, Kruskal-Wallis test $)$, higher vascular invasion status $(P=0.018$, Kruskal-Wallis test; Table 1), and a lower survival rate $(P=0.008$, log-rank test; Figure $2 \mathrm{~B})$. We further compared the CLIC1 levels between the paired primary and metastatic HCC samples from 12 patients (Supstill under debate. CLIC1 is widely upregulated in many types of human cancers (21-26) and is associated with tumor progression and metastasis $(22,27,28)$. However, the involvement of CLIC1 in tumor progress and metastasis has not been fully elucidated.

Here, we demonstrate that CLIC1 is progressively upregulated along with the progression of human hepatocellular carcinoma (HCC) and associated with tumor metastasis. In response to chemotaxic or mechanotaxic stimuli, CLIC1 recruited PIP5Ks to the leading edge of the plasma membrane, where it generates phosphatidylinositol 4,5-bisphosphate ( $\mathrm{PIP}_{2}$ ) and subsequently activation of talin and integrin $\alpha 4 \beta 1$ and $\alpha 6 \beta 4$ to initiate the assembly of nascent cell-matrix adhesions and adhesion-mediated signaling for actin cytoskeleton remodeling to form lamellipodia and invadopodia. CLIC1 deletion suppressed the formation of nascent adhesions and signaling of tumor cells in vitro and tumor metastasis in vivo. Our findings suggest CLIC1 as a potential therapeutic target for cancer invasion and metastasis.

\section{Results}

Comparative proteomics disclosed that CLIC1 is progressively upregulated along with HCC progress. HCC is highly invasive (29). To identify proteins contributing to the high invasiveness of HCC, we compared the protein profiles in 7 normal liver samples, and 12 early (solitary tumor $<3 \mathrm{~cm}$ without invasion), and 13 invasive (invading into major branches of the portal or hepatic veins) HCC samples by using 2D gel electrophoresis (Supplemental Table 1; supplemental material available online with this article; https:// doi.org/10.1172/JCI133525DS1) (Figure 1A). A total of 1238 spots matched across gels and 104 proteins were identified (Supplemental Table 2). Of them, CLIC1 was the only one that was progressively upregulated along with tumor progression (relative levplemental Figure 1B). We found significantly higher CLIC1 levels in the metastatic HCC samples than the primary HCC samples ( $P=0.019, t$ test and $P<0.001$, paired $t$ test; Figure $2 \mathrm{C})$.

To consolidate the above findings, we retrieved an HCC cohort from The Cancer Genome Atlas (TCGA, https://cancergenome. nih.gov/) database. We found that progressive CLIC1 upregulation went along with higher tumor stages ( $P=0.0014$; Figure 2D). Moreover, high CLIC1 expression was associated with lower survival $(P<0.001$; Figure 2E).

Considering that pancreas and lung cancers are highly invasive, we retrieved a cohort of pancreatic ductal adenocarcinoma (PDAC, $n=177$ ) and a cohort of non-small cell lung cancer (NSCLC; $n=513$ ) from TCGA data sets. We found that high CLIC1 expression was significantly associated with lower survival in both cohorts $(P<0.001$ and $P=0.021$ for PDAC and NSCLC, respectively; Figure 2, F and G). Collectively, these findings indicate that CLIC1 is upregulated in HCC, and its upregulation is associated with tumor invasion and metastasis and poor clinical outcomes.

CLIC1 facilitates adherence and extravasation of tumor cells for metastasis in mice. To verify the clinical findings that CLIC1 facilitates tumor invasion and metastasis, we used xenograft tumor assays in mice. We first examined CLIC1 expression in a panel of hepatoma cell lines. Cells with high CLIC1 expression displayed relatively mesenchymal traits and high invasiveness (SK-Hep1 and Mahlavu cells vs. Hep3B and HepG2 cells; Supplemental Figure 2). We then silenced CLIC1 expression by using siRNAs (siCLIC1) or small hairpin RNAs (shCLIC1) in luciferase-transformed SK-Hep1 and Huh7 cells for xenograft tumor metastasis in nude mice. Lung metastasis assays were performed by injection of tumor cells through the tail veins of nude mice. Four hours after the injection, approximately equal amounts of tumor cells targeted to the lungs 
A

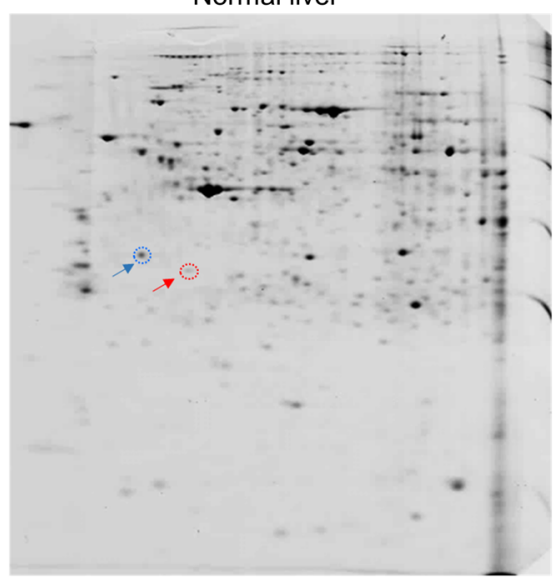

Protein loaded: $150 \mu \mathrm{g}$

Gel condition: $12.5 \%$, nonlinear gel, $17 \mathrm{~cm}$ in length Protein staining: Sypro Ruby

B

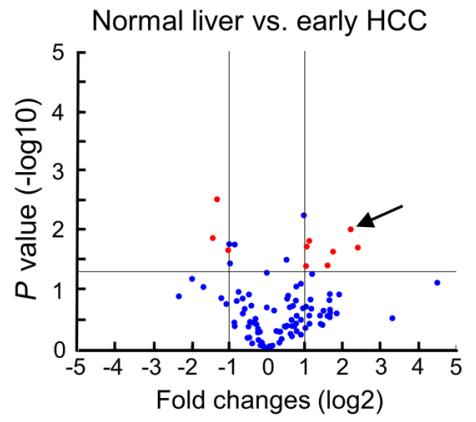

\begin{tabular}{|c|r|r|r|r|}
\hline Ranking & & $\begin{array}{c}\text { Fold } \\
\text { change }\end{array}$ & $\log _{2}$ ratio & $P$ value \\
\hline 1 & VIME & 5.30 & 2.41 & 0.020 \\
2 & CLIC1 & 4.65 & 2.22 & 0.010 \\
3 & ALBU & 3.36 & 1.75 & 0.023 \\
4 & VIME & 3.04 & 1.61 & 0.039 \\
5 & GRP78 & 2.17 & 1.12 & 0.015 \\
6 & TYPH & 2.07 & 1.05 & 0.019 \\
7 & HSP7C & 2.05 & 1.04 & 0.040 \\
\hline 1 & ACDSB & 0.49 & -1.02 & 0.022 \\
2 & ECHM & 0.40 & -1.32 & 0.003 \\
3 & FRIL & 0.37 & -1.43 & $\mathbf{0 . 0 1 4}$ \\
\hline
\end{tabular}
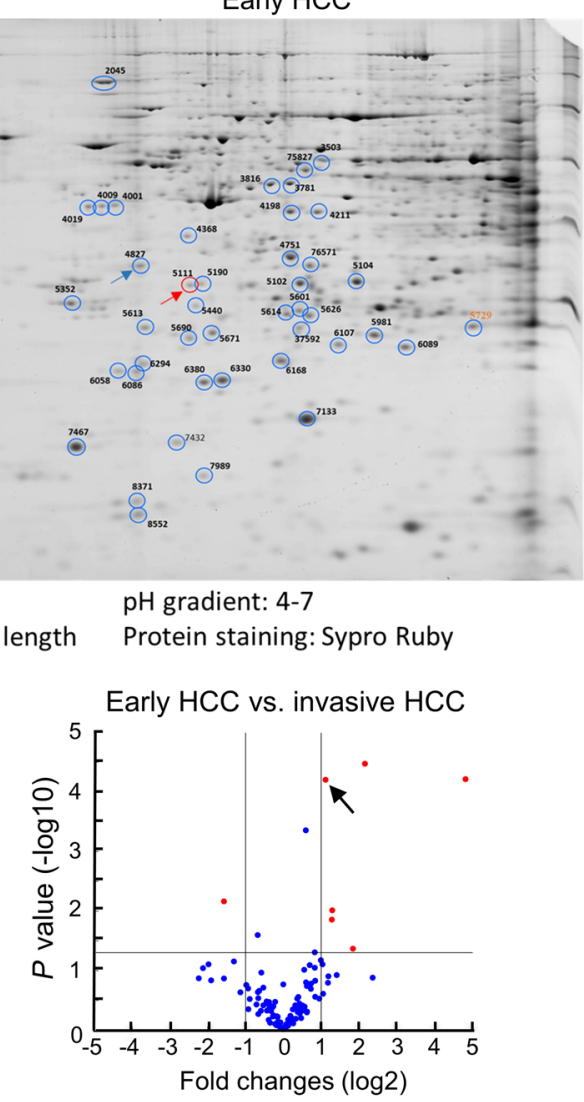

pH gradient: 4-7

Early HCC vs. invasive HCC

\begin{tabular}{|c|r|rrr|}
\hline Ranking & & $\begin{array}{c}\text { Fold } \\
\text { change }\end{array}$ & $\log _{2}$ ratio & $P$ value \\
\hline 1 & FABPL & 28.28 & 4.82 & $\mathbf{0 . 0 0 0}$ \\
2 & PDIA3 & 4.47 & 2.16 & $\mathbf{0 . 0 0 0}$ \\
3 & GDIA & 3.58 & 1.84 & $\mathbf{0 . 0 4 3}$ \\
4 & ACTB & 2.45 & 1.29 & $\mathbf{0 . 0 1 0}$ \\
5 & FRIL & 2.43 & 1.28 & 0.014 \\
6 & CLIC1 & 2.17 & 1.11 & $\mathbf{0 . 0 0 0}$ \\
1 & KHK & 0.34 & -1.57 & $\mathbf{0 . 0 0 7}$ \\
\hline
\end{tabular}

0.014

\section{C}

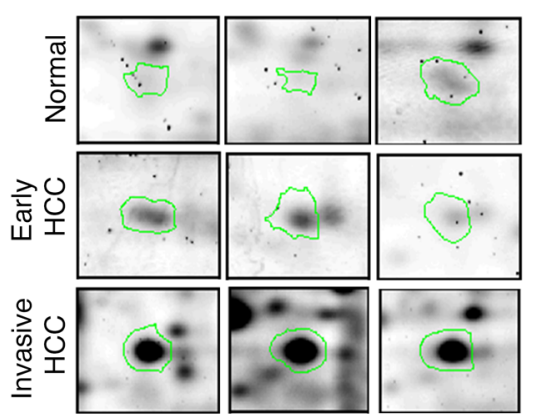

D

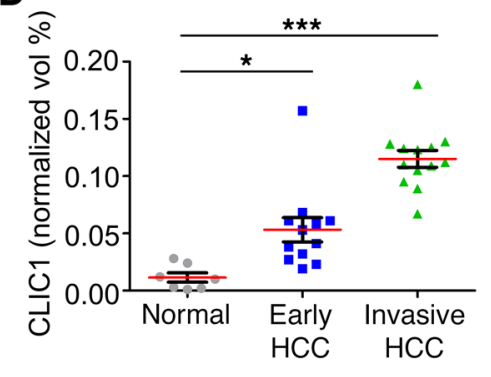

Figure 1. Comparative proteomics identified CLIC1 progressively upregulated along with HCC progress. Liver samples, including 7 normal livers (paratumor liver from cases of hepatic focal nodular hyperplasia), 12 early HCCs, and 13 invasive HCCs, were subjected to proteomics analysis. (A) Representative $2 \mathrm{D}$ gel maps of normal liver, early HCC, and invasive HCC. Red arrows indicate CLIC1 on 2D gel maps. (B) Volcano plots present overviews of the proteins that are differentially expressed in healthy liver, early HCC, and invasive HCC. The $\log _{2}$ fold change and the negative log 10 (FDR) are indicated on the $x$ and $y$ axis, respectively. Proteins with greater than 2-fold changes and $P<0.05$ were regarded as deregulated (red spots) and are summarized in lower panels. Arrows indicate CLIC1, which is the only protein that is progressively upregulated along with HCC progress. Statistical analysis was performed by Mann-Whitney $U$ test. (C) Representative focal 2D images show CLIC1 in normal liver, early HCC, and invasive HCC. (D) Dot plot of the normalized volumes of CLIC1 in normal liver, early HCC, and invasive HCC. Mean \pm SD are shown. Statistical analysis was performed by 1-way ANOVA with Dunnett's corrections. See also Supplemental Tables 1 and $2 .{ }^{*} P<0.05 ;{ }^{* *} P<0.001$. 
A

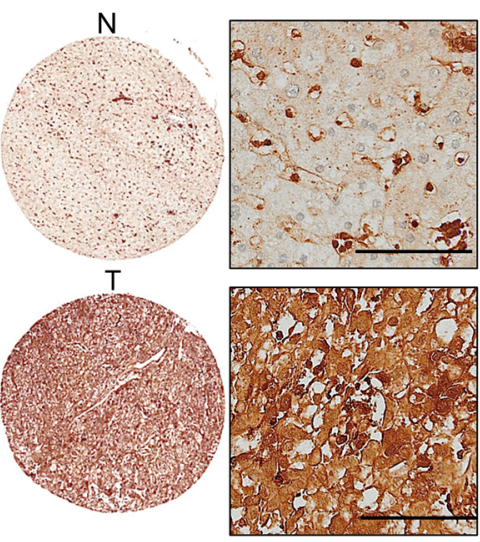

HBV stage III

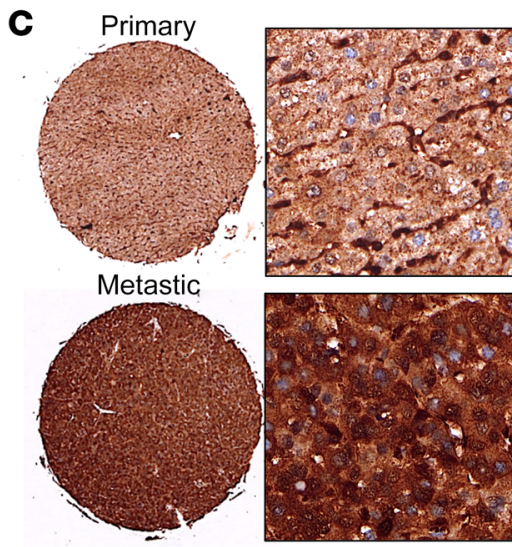

$\mathbf{E}$

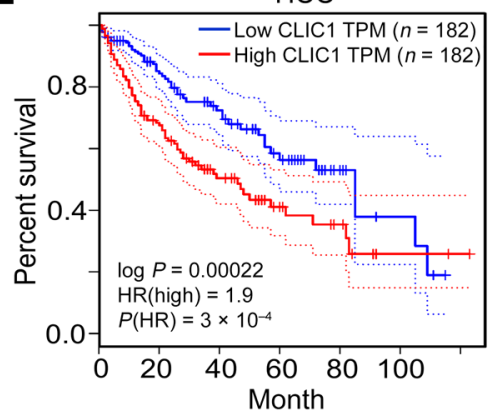

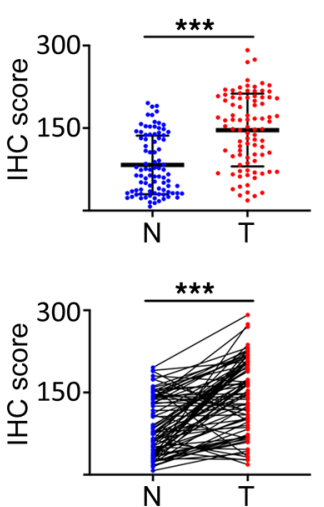
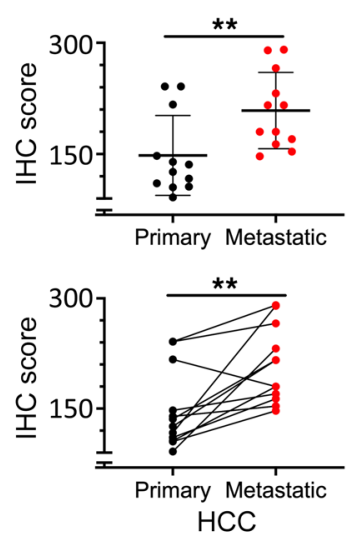

$\mathbf{F}$

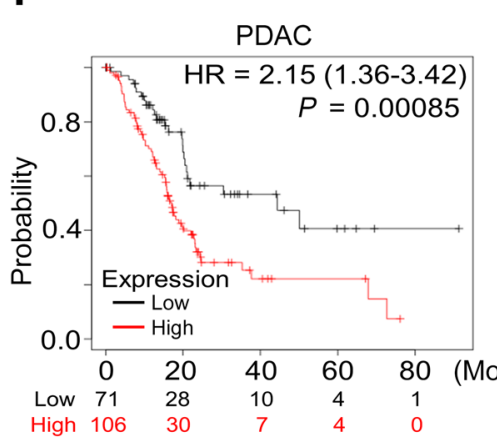

B

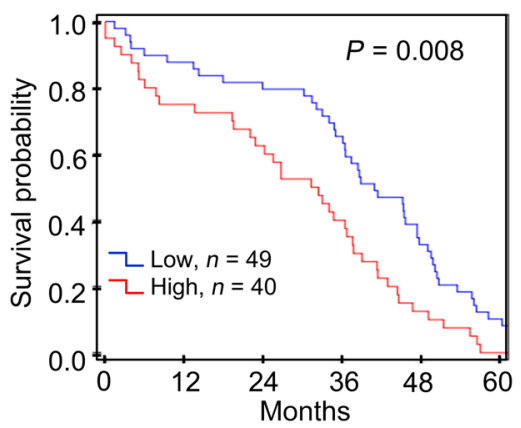

D

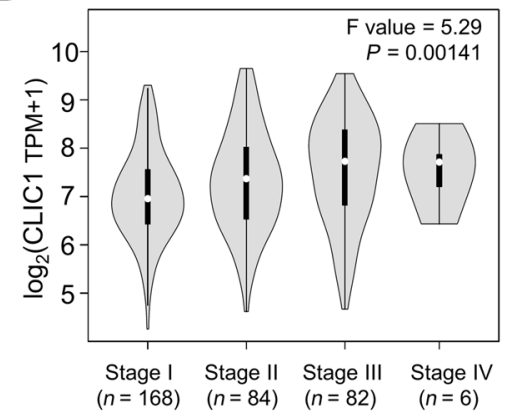

G

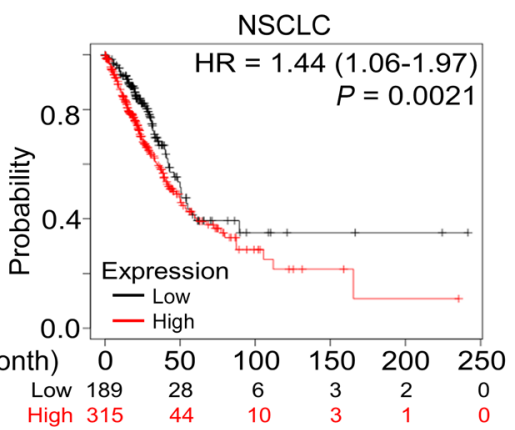

Figure 2. CLIC1 upregulation in HCC is associated with vascular invasion, metastasis, and lower survival. A total of 89 pairs of HCC (T) and para-tumor (N) liver tissues and 12 pairs of primary and metastatic HCCs were included. IHC scores for CLIC1 (calculated as percentage of positive hepatocytes $\times$ IHC intensity [range 0-3]) were determined by an automation system (inForm Advanced Image Analysis Software, version 2.3, PerkinEImer). (A) A pair of representative IHC images of N and T. Scale bar: $100 \mu \mathrm{m}$. Dot plots show the comparison between N and T. Upper, 2-tailed Student's $t$ test; lower, paired $t$ test. ${ }^{* *} P<0.001$. (B) Kaplan-Meier survival curves for the 89 cases of HCC with high (IHC score $\geq 200, n=40$ ) and low (IHC score $<200, n=49$ ) CLIC1 levels. A log-rank test determined $P$ value. (C) Representative IHC images of paired primary and metastatic tumors. Scale bar: $100 \mu \mathrm{m}$. Dot plots: Upper, Mann-Whitney $U$ test; lower, Wilcoxon signed-rank test. ${ }^{* *} P<0.01$. (D) Violin plot shows the relative CLIC1 mRNA levels (central dots: medians; bold bars: interquartile ranges) in different stages of HCC in a TCCA HCC cohort $(n=370)$. Statistical analysis was performed by 1-way ANOVA with Tukey's corrections. TPM, transcripts per kilobase million. (E-G) Kaplan-Meier survival curves generated from 3 TCCA cohorts: HCC $(n=370)$, PDAC ( $n=177)$, and NSCLC $(n=504)$. High and low CLIC1 levels were based on the median CLIC1 value for HCC and the optimal $P$ values by log-rank tests for PDAC and NSCLC.

in all 4 groups were observed (Figure 3A). However, 5-9 weeks after the injection, overt lung metastasis developed in the control groups (siNC, siRNAs containing scrambled sequences; or shEV, shRNA harboring empty vector), but relatively low (siCLIC1) or undetectable (shCLIC1) signals of lung metastasis were found when CLIC1 was transiently (siCLIC1) or constitutively (shCLIC1) silenced (Figure 3, A-D). The silencing efficiency of the inoculat- ed tumor cells was confirmed by immunoblotting assays (Figure $3 \mathrm{E})$. Moreover, constitutively silenced CLIC1 in the xenograft HCC cells prolonged the overall survival of the mice $(P=0.0049$, shEV vs. shCLIC1; Figure 3F), while transient silencing of CLIC1 by siCLIC1 exerted only a marginally protective effect $(P=0.083$; Figure 3G). Consistently, CLIC1 depletion (shCLIC1) suppressed liver metastasis (as compared with the control, shEV), which was 
A

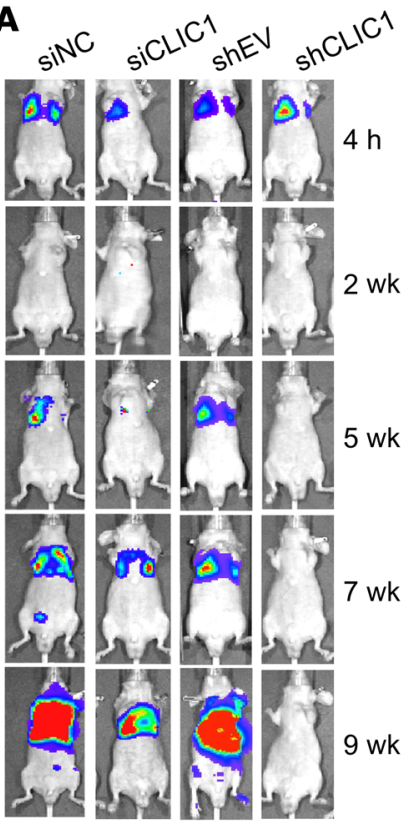

B

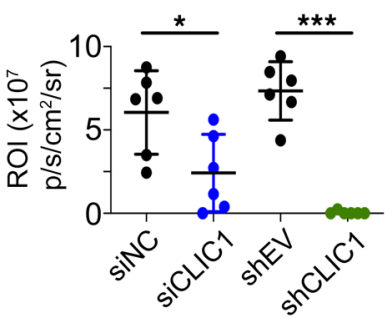

E

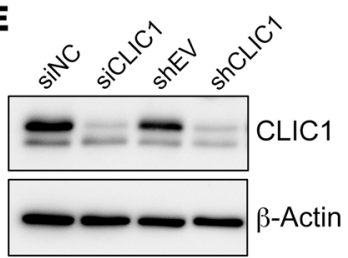

C
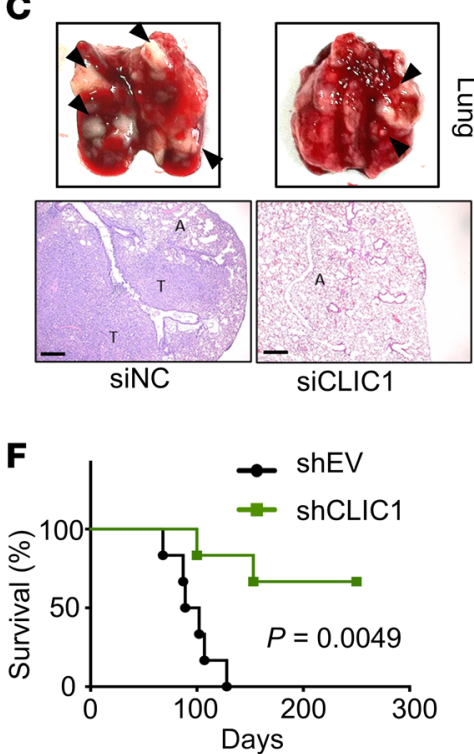

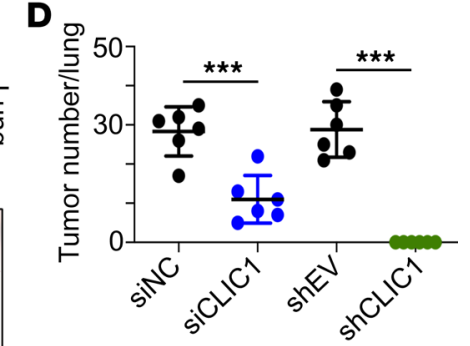

H

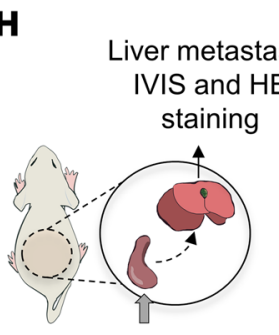

Intrasplenic injection

of cancer cells

(Luc-tagged)

o
I

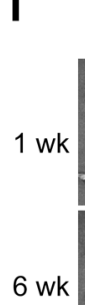

6 wk

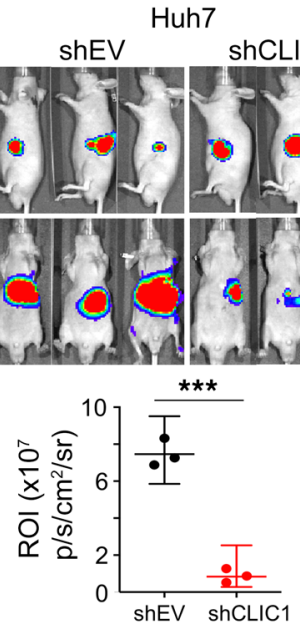

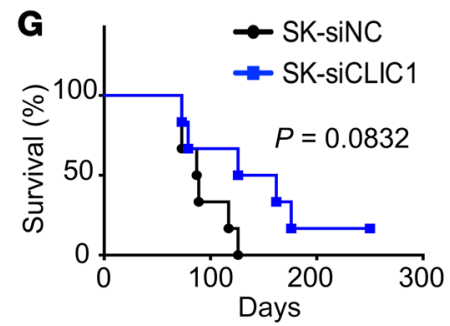

$\mathbf{L}$

Figure 3. CLIC1 facilitates adherence and extravasation of tumor cells for metastasis in mice. Luciferase-transduced SK-Hep1 and Huh7 cells were used for in vivo metastasis in nude mice. Cells transfected with siRNAs containing scrambled sequences (siNC) or targeting CLIC1 (siCLIC1), or constitutively transduced with shRNAs targeting CLIC1 (shCLCI1) or an empty vector (shEV). (A) Lung metastasis assays by injecting SK-Hep1 cells $\left(1 \times 10^{6}\right.$ cells in $200 \mu \mathrm{L} \mathrm{PBS} /$ mouse, $n=6$ ) through tail veins. (B) The statistical results of the luminescence signals for lung metastasis. Statistical analysis was performed by using Mann-Whitney $U$ test between 2 groups ( $n=6$ /group; ${ }^{*} P<$ 0.05 ; ${ }^{* *} P<0.001$ ). (C) Representative lung tissues 63 days after injection. Arrowheads, metastatic tumors. Lower panel: $\mathrm{H} \& \mathrm{E}$ staining for the lung sections. A, lung alveoli; T, metastatic tumors. Scale bar: $120 \mu \mathrm{m}$. (D) Dot blot of metastatic tumor foci per lung. Statistical analysis was performed by using Mann-Whitney $U$ test between 2 groups $\left(n=6\right.$ /group; ${ }^{* *} P<$ 0.001). (E) Western blots for CLIC1 silencing efficiency in HCC cells. (F and $\mathbf{G})$ Cumulative survival curves for the mice bearing xenograft tumors. $P$ values were determined by the log-rank test. (H) Liver metastasis in nude mice by injection of luciferase-transduced Huh7 cells through the spleen. (I) Liver metastasis was inspected by IVIS imaging at weeks 1 and 6 after injection. Lower panel: quantification of luciferase signals in the liver 6 weeks after injection. Statistical analysis was performed by using Mann-Whitney $U$ test ( $n=6 /$ group; $\left.{ }^{* * *} P<0.001\right)$. (J) H\&E staining for liver sections. Scale bar: $120 \mu \mathrm{m}$. (K) Western blots for CLIC1 silencing efficiency. (L) GFP-transduced SK-Hep1 cells with or without CLIC1 depletion were injected through tail veins. Lungs were assayed at 4, 8, 12, 16, 20, and 24 hours after injection. Representative lung section images at 8,16 , and 24 hours after injection are shown. GFP-labeled tumor cells were detected and quantified. Statistical analysis was performed by using Mann-Whitney $U$ test between 2 groups ( $n=8$; NS, no statistical significance; $\left.{ }^{* *} P<0.001\right)$. (M) In vitro cell adhesion assays. The experiments were conducted twice independently. Statistical analysis was performed by using 2-tailed Student's $t$ test between 2 groups $\left(n=8\right.$ /group; $\left.{ }^{* * *} P<0.001\right)$.

performed by injection of Huh7 cells through the spleen into nude mice (Figure $3, \mathrm{H}-\mathrm{K}$ ).

In tail-vein injection mouse models, lung metastasis involves adherence in the lung/extravasation, premetastatic niche formation, and colonization/proliferation of tumor cells in the metastatic cascade (30). To identify which steps of metastasis were inhibited by CLIC1 depletion, we did lung dissection at different time points after injection of the GFP-labeled tumor cells through the tail vein in nude mice. As shown in Figure 3L, equal numbers of tumor cells with (shCLIC1) and without (shEV) CLIC1 depletion were detected in the lung sections at 4 and 8 hours, followed by a rapid loss of the shCLIC1 tumor cells, but not shEV cells, in the lung tissue at 12 hours and after that. This finding suggested a defect in the adherence in the lung alveoli and extravasation steps rather than in the subsequent colonization of tumor cells in the lung metastasis cascade.

To further verify the hypothesis, we seeded SK-Hep1 and Mahlavu cells with and without CLIC depletion on the laminin-coated plates. Cells with CLIC1 depletion significantly lost their adhesion to the plats as compared with the control $(P<$ 0.001 for both SK-Hep1 and Mahlavu cells; Figure 3M). Therefore, CLIC1 is critically required for tumor cells adhering to the extracellular matrix. Together, our findings suggest that CLIC1 plays a pivotal role in the adherence and extravasation of tumor cells in the metastasis cascade.

CLIC1 facilitates filopodia, lamellipodia, and invadopodia upon migration induction. Given that adhesion to the extracellular matrix and membranous protrusions are the fundamental processes for cell migration and tumor invasion and metastasis, we evaluated the roles of CLIC1 in the formation of membrane protrusions. We examined the roles of CLIC1 in cell migration and invasion in vitro. Transwell assays showed that CLIC1 depletion (shCLIC1) significantly suppressed the migration and invasion (with and without Matrigel coating, respectively) of SK-Hep1, Mahlavu, and Huh7 cells (Figure 4, A and B). In contrast, ectopic expression of CLIC1 in Huh7, HepG2, and Tong cells (poorly motile cells) enhanced their migration and invasion (Figure 4, C and D, and Supplemental Figure 3, A and B). The silencing and induction efficiencies of CLIC1 expression are summarized in Supplemental Figure 3C.

Notably, CLIC1 depletion in SK-Hep1, Mahlavu, Hep3B, and Huh7 cells or ectopic expression of CLIC1 in Huh7 and HepG2 cells did not affect cell proliferation (Supplemental Figure 4). Thus, CLIC1 is functionally involved in the regulation of tumor cell migration and invasion in vitro and tumor metastasis in vivo.

We then studied the role of CLIC1 in membrane protrusion in response to migration-related stimuli. We cocultured Huh7 cells with and without CLIC1 silencing on the same plate. As shown in Figure $4 \mathrm{E}$, free-space exposure induced the formation of filopodia in control cells, but not in the cells with CLIC depletion (negative for green fluorescence, Figure $4 \mathrm{E} ; P=0.032$ ). Interestingly, some CLIC1 was targeted to the tip of filopodia. In response to epithelial growth factor (EGF) treatment (chemotaxis), the formation of lamellipodia was induced in the control cells (shEV), but not in cells with CLIC1 depletion (shCLIC1, Figure 4F). Notably, CLIC1 accumulated at the leading edge of lamellipodia was seen (Figure $4 \mathrm{~F}$, rightmost panel). In a time-lapse observation for cell response to EGF treatment, CLIC1-GFP (fusion with GFP) and GFP were initially evenly distributed in the cytosol with spared membrane edges (Figure 4G, 0 min). Then, some CLIC1-GFP, but not GFP, was targeted to the leading edge of the membrane protrusions of migrating cells (Figure 4G). In 3D invasion assays, CLIC1 was targeted to the front end of invading tumor cells (Figure $4 \mathrm{H}$ ), while the invasion was suppressed in the tumor cells with CLIC1 depletion (Figure 4B). Therefore, these findings suggest CLIC1 is targeted to the tips of the membrane protrusions, and plays a crucial role in the formation of filopodia, lamellipodia, and invadopodia for cell migration and tumor invasion.

CLIC1 directs the formation of nascent cell-matrix adhesions and signaling. Since cell-matrix adhesions are the membranous hubs essential for cell adhering to the extracellular matrix and migration, we speculated that CLIC1 might be involved in the formation of nascent cell-matrix adhesions. We examined the formation of cell-matrix adhesions in response to EGF (chemotaxis; Figure 5A) and exposure to free space (wounding, mechanotaxis; Figure 5B). The results showed that CLIC1 was not only targeted to the leading edge plasma membrane but also colocalized with vinculin and talin (Figure 5, A and B, white arrowheads), landmarks of cell-matrix adhesions. In contrast, the lamellipodia formation was substantially suppressed in cells with CLIC1 depletion (Figure 5, A and B, open arrowheads). To further confirm that CLIC1 was targeted to nascent cell-matrix adhesions, we inspected the formation of nascent cell-matrix adhesions by reseeding CLIC1GFP-transfected cells on culture plates. CLIC1 was translocated to the membranous margin 15 minutes after reseeding and then tran- 

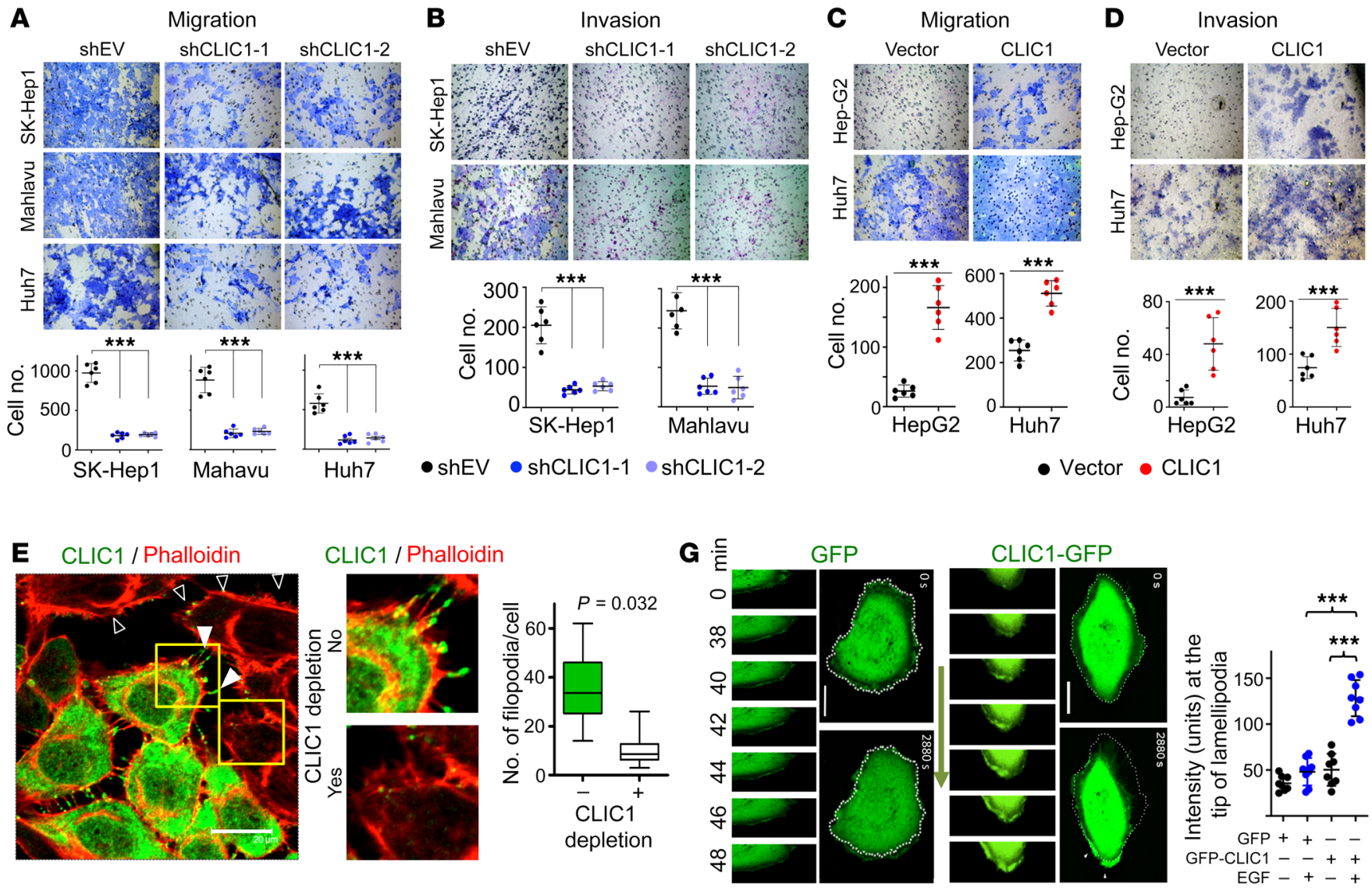

$\mathbf{F}$

EGF $(100 \mathrm{ng} / \mathrm{mL})$

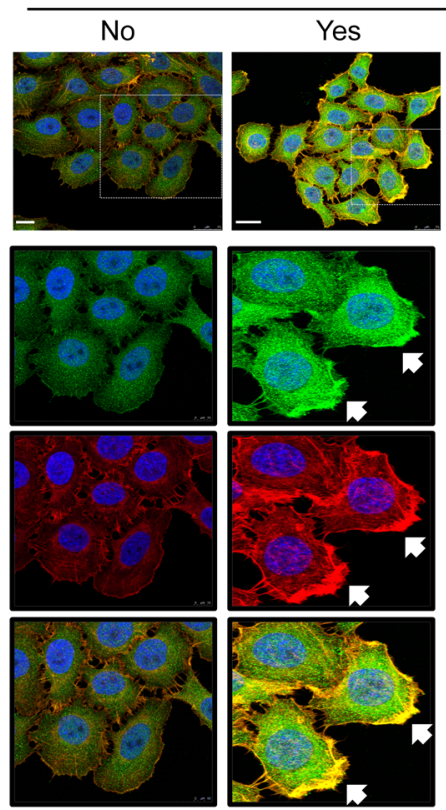

shEV
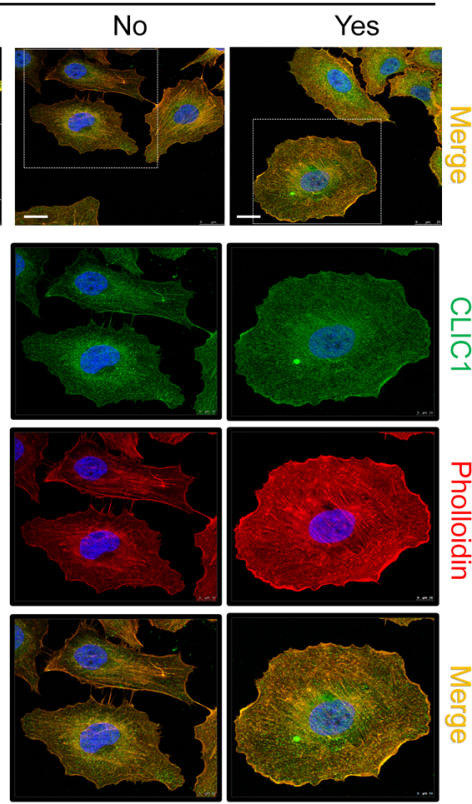

shCLIC1
H
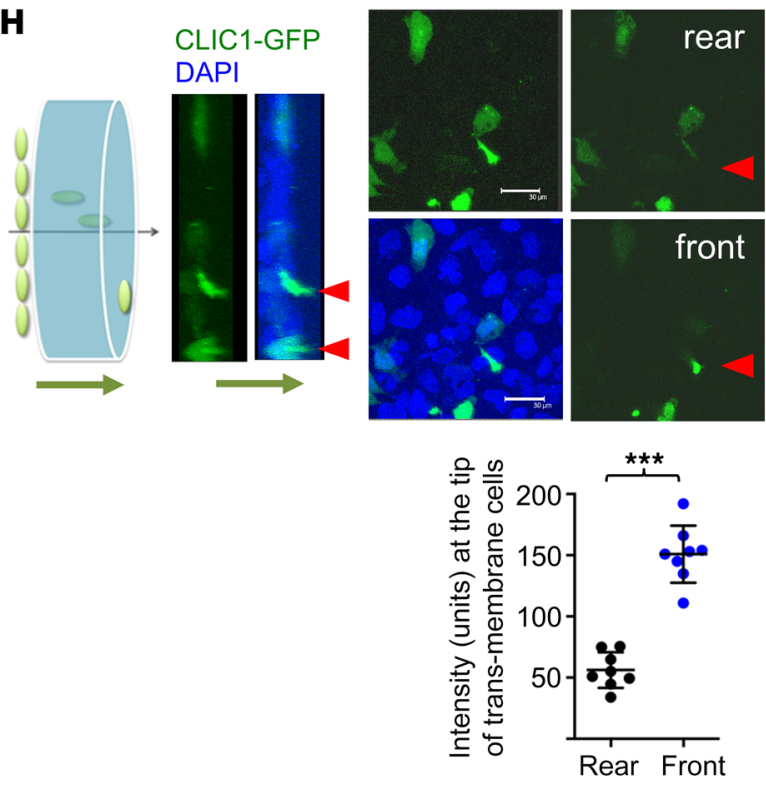

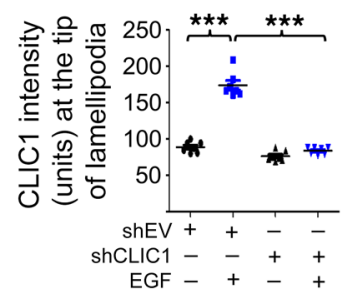


Figure 4. CLIC1 facilitates filopodia, lamellipodia, and invadopodia upon migration induction. (A-D) Transwell assays for migration and invasion (with Matrigel coating) of tumor cells with and without CLIC1 depletion (A and B), or with and without ectopic expression of CLIC1 (C and D). Two shRNA clones targeting CLIC1 mRNA were used. Vector, empty vector. Statistical analysis was performed by using 1-way ANOVA with Dunnett's test (A and B) or 2-tailed Student's $t$ test ( $n=6$ /group in triplicate; ${ }^{*} P<$ $0.01 ;{ }^{* *} P<0.001$ ). See also Supplemental Figure 3. (E) Huh7 cells with (open arrowheads) vs. without CLIC1 depletion 8 hours after free-space exposure. Green (Alexa 488), endogenous CLIC1. Red (Alexa 549), F-actin. White arrowheads: CLIC1 accumulated at the filopodia tips. Box plot: Relative numbers of filopodia per cell. $P$ values were determined by 2 -tailed Student's $t$ test ( $n=20$ cells/group). Scale bar: $20 \mu \mathrm{m}$. (F) Huh7 cells with and without CLIC1 depletion before and 10 minutes after EGF treatment. Arrows: CLIC1 at lamellipodia of migrating cells. Scale bars: $10 \mu \mathrm{m}$. Dot plot: the relative fluorescence intensity at the lamellipodia tips per cell. $P$ values were determined by using 2-way ANOVA with Tukey's corrections ( $n=8$ cells/group). (G) Time-lapse tracking of GFP (upper panel) and CLIC1-GFP (lower panel) in Mahlavu cells in response to EGF treatment. Images were taken every 2 minutes after EGF treatment. Arrow: migration direction. Scale bar: $10 \mu \mathrm{m}$. Statistical analyses were performed by 2-way ANOVA with Tukey's corrections ( $n=8$ cells/group). (H) 3D invasion assays for CLIC1-GFP cells. Arrows: invasion direction. Red arrowheads: the protruding edge of the invading cells. Arrow: migration direction. Dot plot shows the fluorescence intensity (CLIC1-GFP) at the rear versus front tips of cells during passing the matrix gel. Statistical analyses were performed by using 2 -tailed Student's $t$ test ( $n=8$ cells/group).

siently targeted to the edge of membrane protrusions morphologically consistent with nascent cell-matrix adhesions (30 minutes, Supplemental Figure 5A). We then cotransfected cells with CLIC1RFP (red fluorescence protein) and GFP-tagged adhesion marks, including talin, vinculin, and paxillin (Figure $5 \mathrm{C}$ ). The membranous CLIC1 was colocalized with talin, vinculin, and paxillin at the nascent adhesions (Figure 5C, right panel).

In contrast, neither the membrane domain-deleted mutant CLIC1 (dCLIC1-GFP, deletion of aa 2-90) nor GFP was targeted to the nascent cell-matrix adhesions (Figure 5D). Moreover, the formation of nascent cell-matrix adhesions was also decreased in cells with CLIC1 depletion (Figure 5, A and B). These findings suggested that CLIC1 is required for the assembly of nascent cell-matrix adhesions, which is dependent on the membrane-associated domain of CLIC1.

To further verify the role of CLIC1 in the assembly of nascent cell-matrix adhesions, we used coimmunoprecipitation assays to inspect the integrity of cell-matrix adhesions between the cells with and without CLIC1 depletion. We found that CLIC1 depletion slightly decreased the levels of talin, vinculin, and Src, but not $\beta$-actin, FAK, or caveolin1 (Figure 5E). However, the silencing of CLIC1 decreased the vinculin, FAK, Src, $\beta$-actin, and caveolin levels in the precipitate by using anti-talin antibodies (Figure $5 \mathrm{~F}$, left panel); decreased the vinculin, Src, and $\beta$-actin levels in the precipitate by using anti-FAK (Figure 5F, middle panel); decreased the FAK and $\beta$-actin levels in the precipitate by using anti-Src (Figure $5 \mathrm{~F}$, right panel); and reduced the vinculin, FAK, and Src levels in the precipitate by using anti- $\beta$-actin (Supplemental Figure $5 \mathrm{~B}$ ). The results are summarized in Figure 5G. Collectively, silenced CLIC1 expression disrupts the integrity of cell-matrix adhesions. Indeed, in CLIC1-depleted cells, the formation of nascent cell-matrix adhesions at the leading edge of migrating cells was substantially suppressed (Figure 5, A and B; open arrowheads indicate cells with CLIC1 depletion and no nascent adhesion formed).

If CLIC1 facilitates the assembly of nascent cell-matrix adhesions, we predicted that CLIC1 promotes the nascent adhesion-mediated signaling. We compared adhesion-mediated cellular signaling between inactive (no nascent adhesion formation) or reseeded (inducing nascent adhesions) cells. We found that CLIC1 depletion only suppressed the phosphorylation of FAK, Src, and AKT in reseeded cells but not in inactive cells (Figure $5 \mathrm{H}$, left panel). Moreover, CLIC1 depletion selectively suppressed the ITGB4 and ITGA4 levels as well as the phosphorylation of Src and AKT in the reseeded cells but not in inactive cells (Figure 5H, right panel), suggesting that CLIC1 is selectively involved in the regulation of nascent, but not mature, adhesion-mediated signaling.

Overall, these findings support the hypothesis that CLIC1 is specifically required for the induction of nascent cell-matrix adhesions and signaling, which is known to drive F-actin elongation for lamellipodia and filopodia formation (31).

CLIC1 recruits PIP5K to the plasma membrane to initiate PIP $/$ talin/integrin-mediated cell-matrix adhesion formation. To further investigate the mechanism by which CLIC1 facilitates nascent adhesion formation, we used IP and found that PIP5K1A and PIP5K1C were bound to CLIC1 (Figure 6, A and C). Specific binding of PIP5K1A and PIP5K1C with CLIC1 was further confirmed by reciprocal IP assays (Figure 6B) or competition binding assays with a recombinant CLIC1 (Figure 6D).

We then inspected the interaction of PIP5K1A and PIP5K1C with CLIC1 at the subcellular level. We found that EGF induced cotargeting of CLIC1 and PIP5K1A to the leading edge of membrane protrusions (Figure 6E). Consistently, free-space exposure induced cotargeting of CLIC1 and PIP5K1A to the leading edge of lamellipodia in 2 primary HCC cell lines derived from patients with HCC (Supplemental Figure 6). However, in response to EGF treatment, not only the membrane-targeting of PIP5K1A but also the formation of membrane protrusions were substantially reduced in the cells with CLIC1 depletion (Figure 6F; open arrowheads) as compared with cells without CLIC1 deletion (Figure 6F; arrowheads). Consistently, CLIC1 depletion suppressed accumulation of PIP5K1C to the plasma membrane and colocalization with nascent cell-matrix adhesions in reseeding assays (Figure $6 \mathrm{G}$, red arrowheads [shCLIC1] vs. yellow arrowheads [shEV]).

To further confirm that CLIC1 is required for translocation of PIP5K1A/C from the cytosol to the plasma membrane, we compared the PIP5K1A levels associated with the plasma membrane and in the cytosol in response to EGF treatment. EGF treatment increased the membranous PIP5K1A by 2.5 fold, but not the cytosolic PIP5K1A, in cells without CLIC1 depletion, consistent with membrane translocation of PIP5KIA (Figure $6 \mathrm{H}$, lanes $1,2,5,6)$. However, in cells with CLIC1 depletion, not only was the membranous PIP5K1A level substantially decreased but also the response to EGF treatment was abolished (Figure 6H, lane 3,4). Therefore, CLIC1 is required for the recruitment of PIP5K1A to membrane. As a result, our data support the hypothesis that, in response to chemotaxis or mechanotaxis (free-space exposure), the membrane-targeted CLIC1 recruits PIP5K1A/C to the plasma membrane to facilitate the formation of nascent cell-matrix adhesions. 

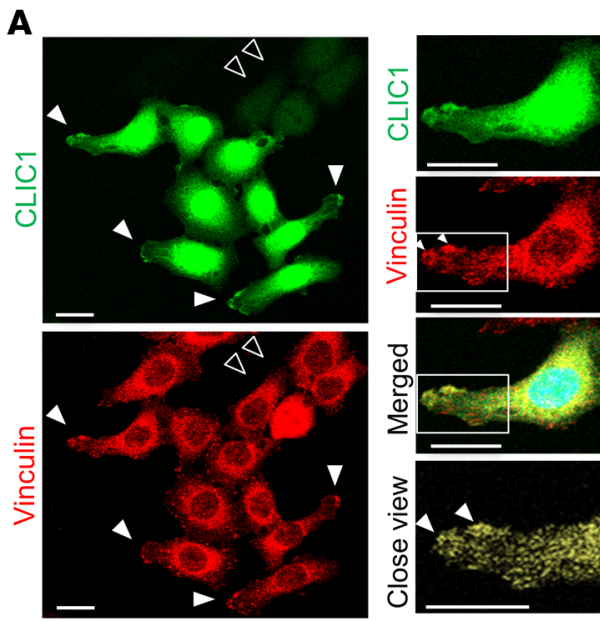

siNS

C

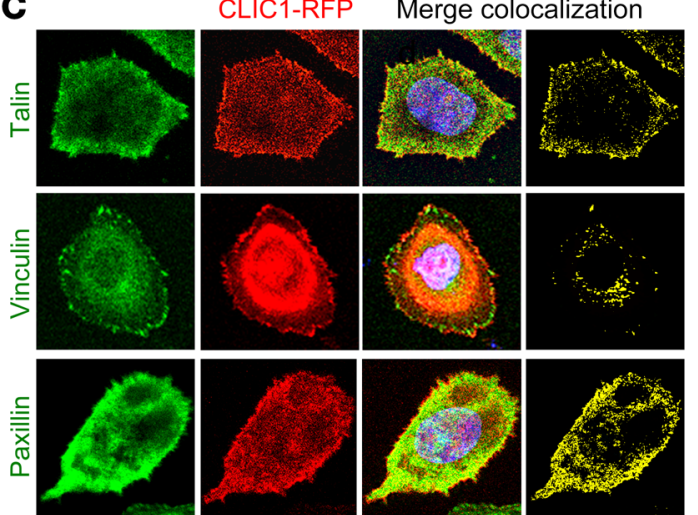

E

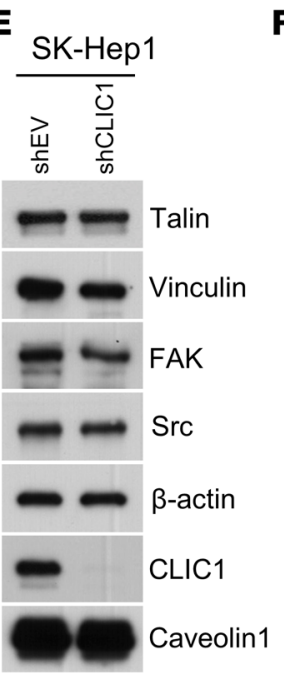

$\mathbf{F}$
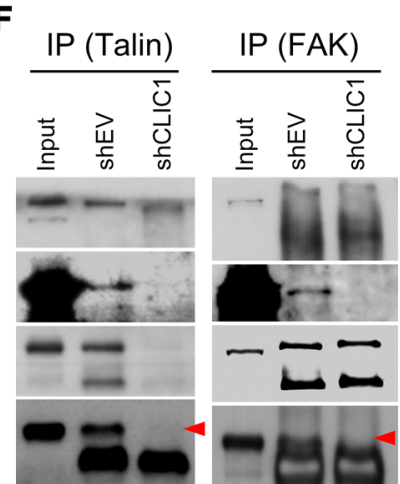
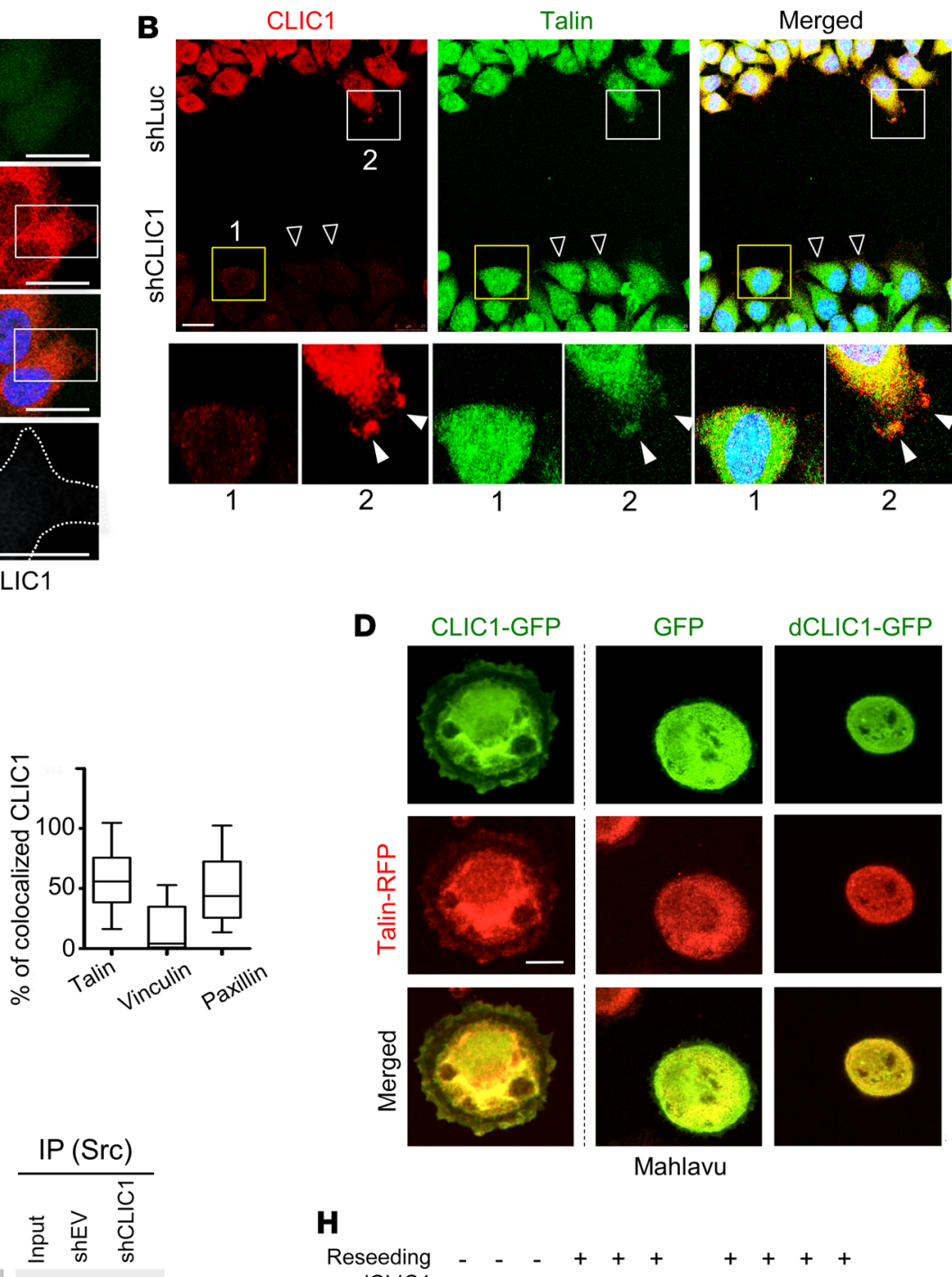

CLIC1

G
(c) $\mathrm{T}$
(C) $\mathrm{V}$
(T) Talin
(V) Vin
(A) $(F)$
(S)
(A) $(\mathrm{S}) \mathrm{F}$
(F) FAK
(S) Src
(A) Actin
shEV
(C) Cav1

D
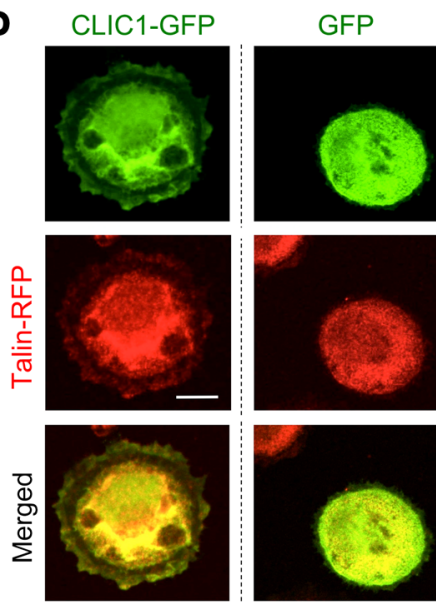

Mahlavu

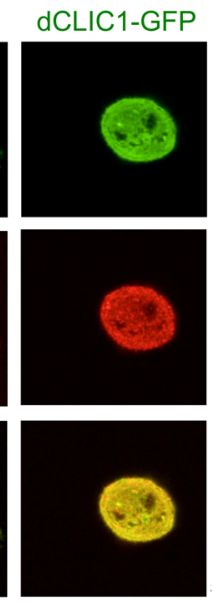

H

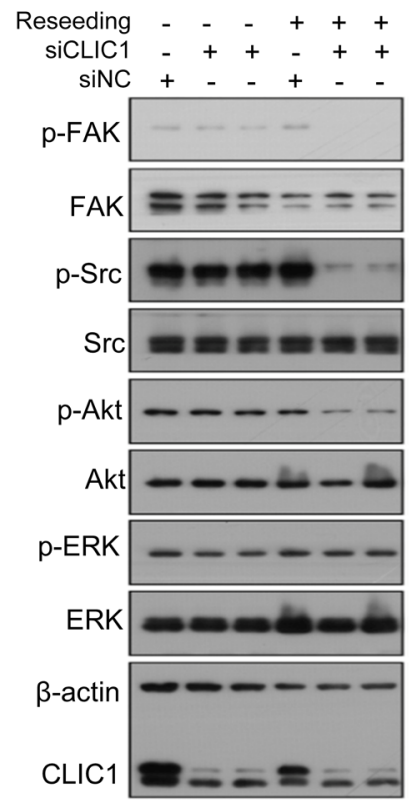

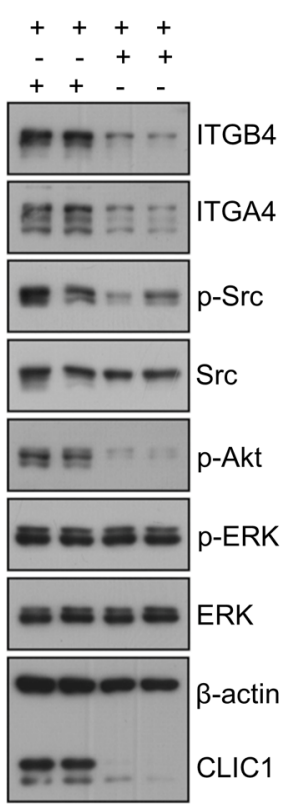


Figure 5. CLIC1 directs the formation of cell-matrix adhesions and signaling. (A and B) Nascent cell-matrix adhesions in lamellipodia of cells with and without CLIC1 depletion in response to (A) EGF treatment (10 minutes, $100 \mathrm{ng} / \mathrm{mL}$ ) or (B) exposure to free space (8 hours after wounding). White arrowheads indicate the tips of lamellipodia. Open arrowheads indicate cells with CLIC1 depletion, in which few lamellipodia and nascent adhesions were seen. Scale bar: $10 \mu \mathrm{m}$. (C and $\mathbf{D})$ Confocal images for the formation of nascent cell-matrix adhesions 15-30 minutes after cells reseeded on laminin-coated plates. See also Supplemental Figure 5A. (C) Red, CLIC1-RFP; green, talin-GFP, vinculin-GFP, and paxillin-GFP. Box plots: the median and interquartile ranges of the amounts of CLIC1 colocalized with talin, vinculin, and paxillin. (D) Colocalization of CLIC1-GFP but not dCLIC1 (deleted transmembrane domain) or GFP with talin at nascent cell-matrix adhesions. Scale bar: $5 \mu \mathrm{m}$. (E) Immunoblots show the effects of silencing CLIC1 on the talin, vinculin, FAK, Src, and actin levels. (F) Coimmunoprecipitation assays using antibodies against talin, FAK, and Src to pull down binding proteins of SK-Hep1 cells with versus without CLIC1 depletion. Immunoblots with antibodies to detect the key components of cell-matrix adhesions. See also Supplemental Figure 5B. Each assay was performed independently at least twice. (G) Cartoons summarize the results from co-IP. (H) CLIC1 selectively required for activation of nascent adhesionmediated signals. Huh7 cells transfected 2 different clones of siCLIC1 or a control siRNA (siNC). We compared the cells after they had been reseeded $(+)$ or in inactive status (-). Reseeding assays were used to induce the formation of nascent cell-matrix adhesions.

It is known that talin can be activated by PIP ${ }_{2}$, which is a product of PIP5Ks, resulting in the assembly of integrin-mediated adhesions $(32,33)$. We thus tested whether the membrane-targeting of CLIC1 facilitated PIP $_{2}$ accumulation in the plasma membrane for nascent cell-matrix adhesions. As shown in Figure 6I, in the reseeded cells, $\mathrm{PIP}_{2}$ was highly accumulated at the edge of the plasma membrane (left panel) where nascent cell-matrix adhesions were formed. In contrast, CLIC1 depletion suppressed the accumulation of PIP in the plasma membrane (right panel).

Collectively, the above findings support the hypothesis that CLIC1 recruits PIP5Ks to the plasma membrane to activate the $\mathrm{PIP5K} / \mathrm{PIP}_{2} /$ talin/integrin signaling pathway to induce nascent adhesions and adhesion-mediated signals. As such, ectopic expression or activation of PIP5Ks or talin would restore the nascent adhesion formation and signals in the cells with CLIC1 depletion. Consistently, ectopic expression of PIP5K1C or talin prevented the suppression of nascent adhesion-mediated signaling (phosphorylation of FAK, paxillin, and Src) and integrins $\alpha 4$ and $\beta 4$ by CLIC1 depletion (Figure $6, \mathrm{~J}-\mathrm{L}$ ).

The expression levels of adhesion-related effectors predict poor clinical outcomes in HCC. Given that cell-matrix adhesions play a crucial role in tumor invasion and metastasis, we hypothesized that the cell-matrix adhesion markers might predict clinical outcomes, as does CLIC1 in patients with HCC. We retrieved a cohort of 370 patients with HCC from the TCGA database (34). We found a positive correlation of the CLIC1 expression levels with the cell-matrix adhesion markers, including talin $\left(P=5.6 \times 10^{-10} ; R=\right.$ $0.32)$, vinculin $\left(P=2.2 \times 10^{-12} ; R=0.35\right)$, paxillin $(P=0 ; R=0.49)$, and FAK $\left(P=2.8 \times 10^{-11} ; R=0.34\right.$; Figure 7A). Interestingly, high expression levels of talin $(\mathrm{HR}=1.44 ; P=0.039)$, vinculin ( $\mathrm{HR}=$ $1.74 ; P=0.0027)$, paxillin $(\mathrm{HR}=1.79 ; P=0.0017)$, and FAK (PTK2; $\mathrm{HR}=1.49 ; P=0.030)$ in HCC were associated with a high mortality rate (Figure 7B). These findings further support the implication of cell-matrix adhesions in HCC progression.
CLIC1 and its ion conductance are therapeutic targets for tumor metastasis. Given that membranous CLIC1 can function as a chloride ion channel $(17,20,35)$ and is associated with tumor invasion and metastasis, we speculated that CLIC1 or its chloride conductance are potential therapeutic targets for tumor metastasis. To test this hypothesis, we treated tumor cells with indanyloxyacetic 94 (IAA-94), a small molecule that is known to inhibit CLIC family channel $(35,36)$. We found that IAA-94 interfered with tumor cell adhesion to the laminin-coated culture plates compared with those treated with dimethyl sulfoxide (DMSO, the solvent) as a control (Figure 8A), similar to the suppression of cell adhesion by CLIC1 depletion (Figure 3M). We then injected luciferase-transduced SK-Hep1 cells through tail veins of nude mice to assay lung metastasis. Equal numbers of tumor cells were trapped in the lungs 3 hours after injection (Figure 8B). However, IAA-94 treatment or constitutive silencing of CLIC1 significantly suppressed the subsequent lung metastasis ( 50 days after injection; $P<0.001$, shEV vs. shCLIC1; $P<0.001$, DMSO vs. IAA-94). We dissected lung tissues (Figure $8 \mathrm{C}$ ) and confirmed the suppression of lung metastasis either by silencing CLIC1 expression with shRNAs (17.4 vs. 0 per lung, $P<0.001)$ or IAA- 94 treatment $(19.8$ vs. 0 per lung, $P<0.001$; Figure 8 C, right panel).

Overall, our study supports the hypothesis that in response to chemotactic or mechanotactic stimuli, CLIC1 recruits PIP5K1A/C to the leading edge of the plasma membrane, where PIP5K1A/C generate a $\mathrm{PIP}_{2}$-rich microdomain to activate talin $(37,38)$. The activated talin then induces the formation of integrin-mediated adhesions and signals for lamellipodia/invadopodia formation in a spatiotemporal regulatory manner (Figure 8D). Aberrant CLIC1 upregulation, which is frequently found in metastatic tumors, endows tumor cells with high motility and invasiveness. Inhibition of CLIC1 or its ion conductance are promising approaches for the prevention and treatment of tumor invasion and metastasis.

\section{Discussion}

Efficient membrane protrusion and adhering to the extracellular matrix are fundamental cell processes and crucial for migration, in particular, in tumor invasion and metastasis. In this study, we identified CLIC1 that is upregulated in human HCC and associated with tumor invasion, metastasis, and poor clinical outcomes. We further demonstrated that, in response to chemotaxis and mechanotaxis, CLIC1 is targeted from the cytosol to the leading edge of the plasma membrane to form both nascent cell-matrix adhesions and the signals for lamellipodia and invadopodia formation in a spatiotemporal regulatory manner. Our findings provide a clue to the answer to a long-term enigma of how tumor cells efficiently regulate lamellipodia and invadopodia for invasion and metastasis.

CLIC1 shuttles between the cytosol and plasma membrane through conformational changes (17) without known biological significance (39). We herein report that in response to chemotaxis by EGF or mechanotaxis by disrupting cell-cell contacts (temporal factors), PIP5K1A/C are recruited by CLIC1 to the leading edge of the plasma membrane, where PIP5K1A/C generate a $\mathrm{PIP}_{2}$-rich microdomain (spatial factors). It is known that PIP $_{2}$ clusters activate talin by way of a pull-push mechanism (38), resulting in inducing integrin-mediated cell-matrix adhesion formation $(37,40)$. Therefore, the shuttling of CLIC1 between the cytosol and plasma 


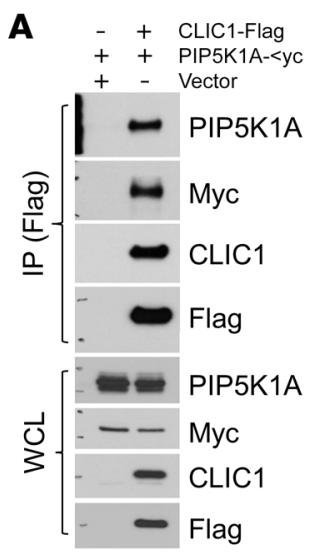

B - + PIP5K1A-Flag

++ CLIC1-RFP
+- Vector

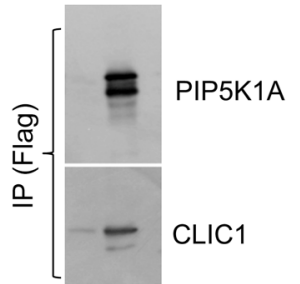

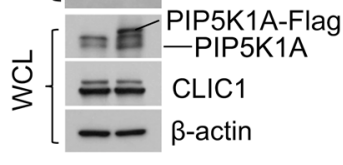

$\mathbf{F}$
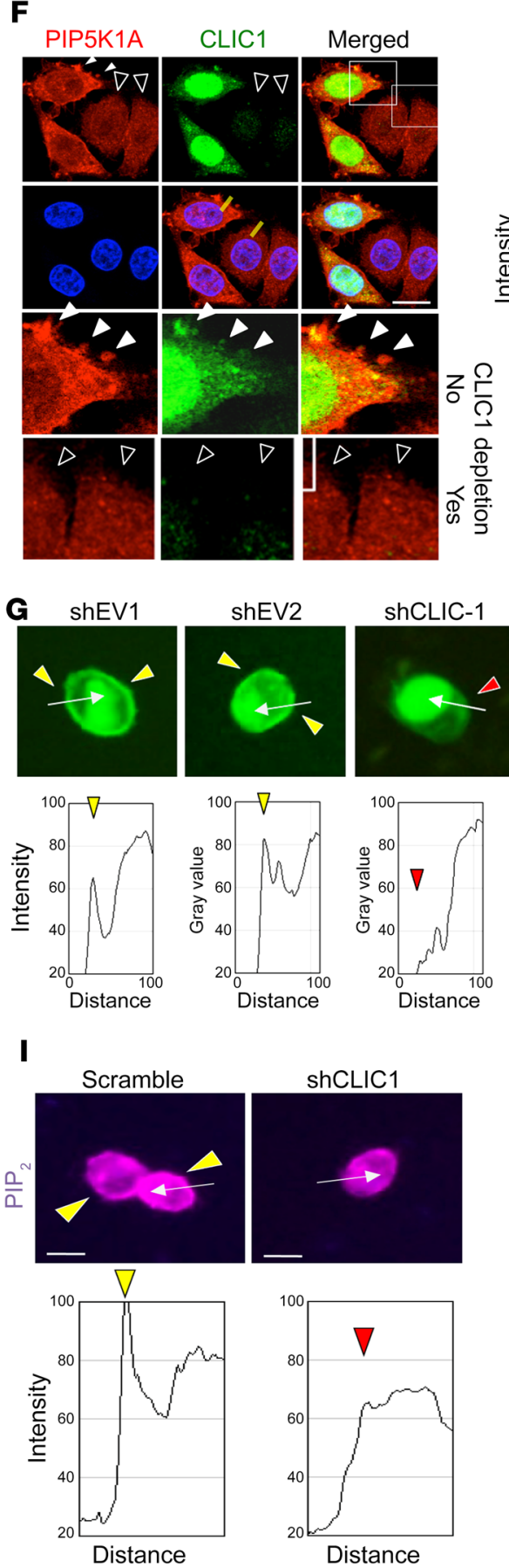
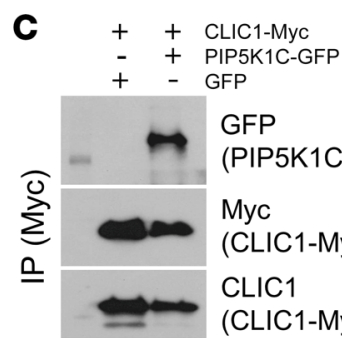

GFP
(PIP5K1C-GFP)

Myc

(CLIC1-Myc)

CLIC1

(CLIC1-Myc)
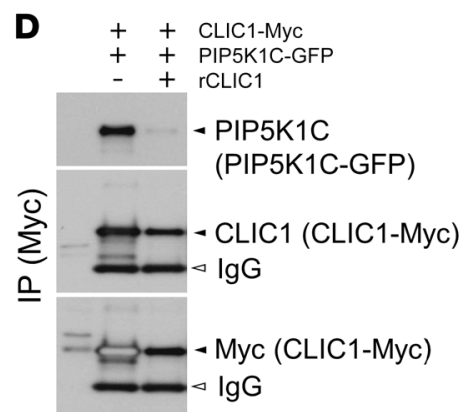

E

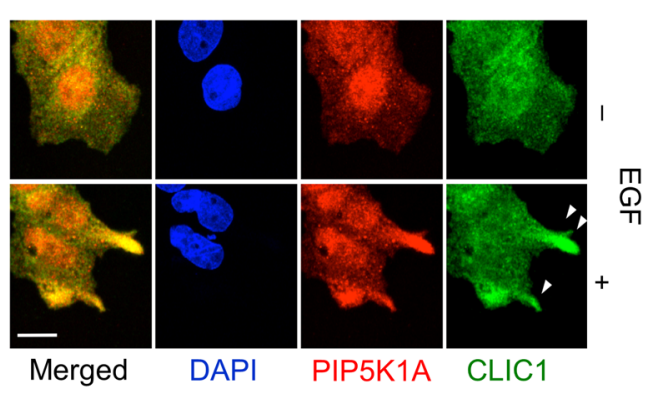

$\stackrel{m}{\mathrm{Q}}$

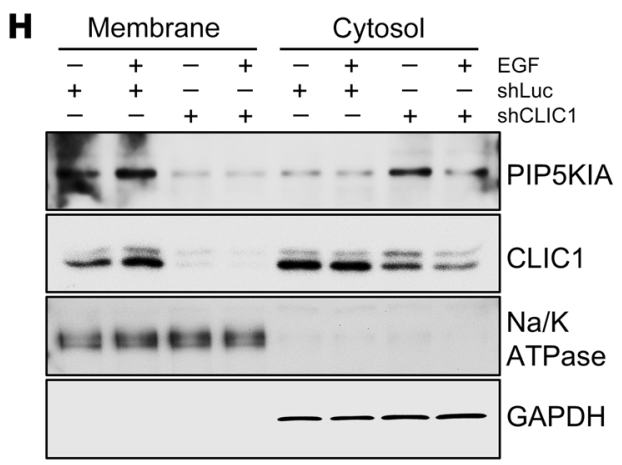

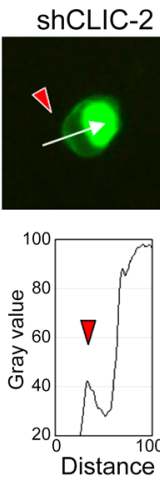
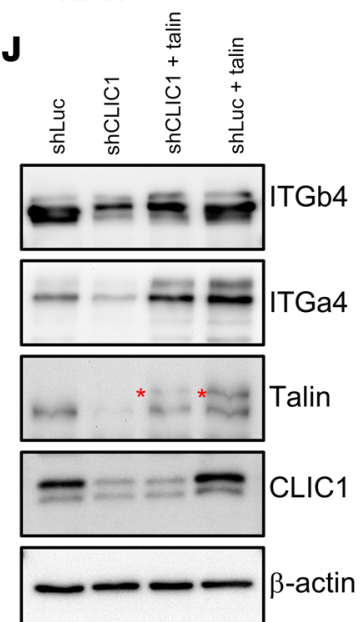

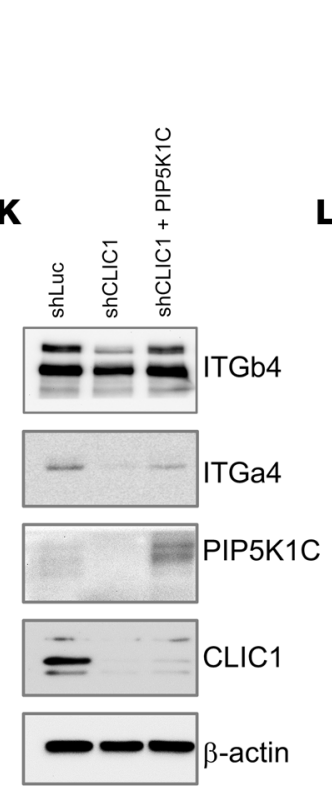


Figure 6. CLIC1 recruits PIP5K to the plasma membrane to initiate PIP $/$ talin/integrin-mediated cell-matrix adhesion formation. (A-D) Immunoprecipitation (IP) by using antibodies against the flag- or myc-tag. WCL, whole-cell lysate as the loading control. (A) IP for CLIC1-bound proteins. (B) IP for PIP5K1A-bound proteins. (C) IP for CLIC1-bound proteins. (D) The binding specificity was examined by a recombinant CLIC1 (rCLIC1) before IP. (E) Immunofluorescence (IF) demonstrates cotargeting of CLIC1 (Alexa 594, green) and PIP5K1A (Alexa 488, red) to the tips of lamellipodia in Huh7 cells before and after EGF treatment ( 10 minutes, $100 \mathrm{ng} / \mathrm{mL}$; indicated by arrowheads). (F) IF for lamellipodia formation and colocalization of PIP5K1A with CLIC1 at the front edge of lamellipodia of cells with versus without CLIC1 depletion after ECF treatment. Scale bars: $10 \mu \mathrm{m}$. (C) Confocal images show CLIC1-dependent accumulation of PIP5K1C at the nascent cell-matrix adhesions of cells after being reseeded. Lower panel: Fluorescence intensities recorded along the arbitrary lines across the cell membrane. (H) Subcellular fractionation for membrane-associated versus cytosolic proteins. $\mathrm{Na} / \mathrm{K}$ ATPase was used as a membranous marker, GAPDH, a cytosolic marker. EGF treatment: $100 \mathrm{ng} / \mathrm{mL}$ for 10 minutes. (I) GFP-PH-PLC, a PIP ${ }_{2}$ biosensor, was used to measure the intracellular concentration of $\mathrm{PIP}_{2}$ (54). Scale bars: $10 \mu \mathrm{m}$. The fluorescence intensities were recorded along the arbitrary lines across the plasma membrane. (J-L) Ectopic expression of talin (J) or PIP5K1C (K and $\mathbf{L}$ ) prevented the suppression of integrins $\alpha 4$ and $\beta 4$ ( $\mathbf{J}$ and $\mathbf{K}$ ) and the nascent adhesion-mediated signals (L) in CLIC1-depletion cells 45 minutes after reseeding.

membrane spatiotemporally regulates the formation of filopodia, lamellipodia, and invadopodia for directional cell migration and tumor invasion and metastasis.

The ability of CLIC1 to shuttle between the cytosol and plasma membrane and its involvement in the regulation of cell adhesion to the extracellular matrix are reminiscent of those found for other CLIC family members. Argenzio et al. found that CLIC4 is rapidly recruited to the plasma membrane and colocalized with $\beta 1$ integrin in response to the activation of RhoA-mediated signaling $(41,42)$. Moreover, CLIC4 is critical for $\beta 1$ integrin internalization and recycling. Interestingly, the membrane-targeting of CLIC4 provides a feedback mechanism to counteract filopodium formation (41). CLIC3 is upregulated and required for the invasion of breast and pancreatic cancers by regulating Rab25-dependent a5 31 integrin recycling (43) and Rab25-independent MMP14 recycling (44). Apparently, different CLIC members have different cellular functions but share similar molecular mechanisms.

Since CLIC1 is upregulated in many types of human cancers and selectively targeted to the plasma membrane in tumor cells, it can be a promising target for the anticancer treatment of invasion and metastasis. Notably, the CLIC1 gene is highly conserved among tumors, with only $2 \%$ of patients harboring missense or nonsense mutations, indicating that its role in tumorigenesis is more related to its membrane localization-associated activity than to mutations (45). Gurski et al. reported that in renal cell carcinoma, CLIC1 cooperates with integrin $\alpha v \beta 3$ and fibronectin to stabilize invadopodia for tumor invasion by regulating myosin light chain kinase (MYLK) (46). Recently, it was reported that silencing CLIC1 impairs the proliferative capacity and self-renewal properties of glioblastoma cells, thereby indicating the vital role of CLIC1 in sustaining the stemness of cancer cells. Given that cancer cells with chemo-resistance and metastasis have been attributed to a minor fraction of tumors with stemness properties, targeting CLIC1 provides a novel approach to directly eradicate such cancer stem cells
$(47,48)$. Further studies to evaluate the efficacy of anti-cancer therapy by targeting CLIC1 in human cancers are warranted.

In summary, membrane targeting of CLIC1 regulates nascent cell-matrix adhesions, signaling, and membrane protrusions by way of transporting PIP5Ks to the plasma membrane. This CLIC1/ $\mathrm{PIP5K} / \mathrm{PIP}_{2} /$ talin/integrin/adhesion signaling pathway orchestrates the spatiotemporal formation of lamellipodia and invadopodia for tumor invasion and metastasis. Our findings suggest that CLIC1, with its unique properties in cancer cells, can serve as an excellent target for the prevention and treatment of invasion and metastasis in cancers.

See complete unedited blots in the supplemental material.

\section{Methods}

See supplemental material for additional details.

Cell lines, cDNA clones, siRNAs, shRNAs, and reagents. We used Huh7, Hep3B, Mahlavu, and HepG2 (human hepatoma cell lines); SK-Hep1 (a human hepatic adenocarcinoma cell line); and 2 primary HCC cell lines, LT87 and PDX57, which were derived from 2 patients with HCC, in this study. For immunoprecipitation assays, we used human 293T cells because of their high efficiency in the expression of transduced clones. The cells were cultured in DMEM (Thermo Fisher Scientific) with 10\% FBS. Plasmid pCLIC1-GFP was from Origene (\#RG218042), and plasmids pPIP5K1A-Myc (\#20580), pPIP5K1C-GFP (\#22299), and PLC-delta-GFP were from Addgene (\#21179). The PIP5K1A cDNA, which was cloned into pCMV6-Myc, was from Addgene (\#20580). pEGFP-C2-PIP5K1C90 was from Addgene (\#22299). The target sequences of siRNA or shRNA for CLIC1 were TGGCTCAAGGAGTCACCTTCAATG and CCCATTCCTGCTGTATGGCACTGAA (Thermo Fisher Scientific). The MQAE staining reagent was from Thermo Fisher (catalog E3101). $\mathrm{R}(+)$-IAA-94 was purchased from MilliporeSigma (catalog I117). Pseudolentivirus for shRNAs targeting CLIC1 mRNA (shCLIC1), luciferase (shLuc), or an empty vector (shEV) were obtained from National RNAi Core. Overexpression of CLIC1 was performed using the Lenti-X Tet-Off Advanced Inducible expression system (632163; Clontech) according to the manufacturer's instructions.

Comparative proteomics. For 2D proteomics, we collected tumor and para-tumor liver tissues from 7 patients with hepatic focal nodular hyperplasia, 12 patients with early HCC (solitary tumor $<3 \mathrm{~cm}$ without vascular invasion), and 13 patients with invasive HCC (with invasion into main branches of the portal or hepatic veins) who received hepatectomy in our hospital. 2D gel electrophoresis, gel staining, image analysis, and protein identification were performed as previously described (49).

In vivo metastasis assays. We used SK-Hep1 and Huh7 cells for in vivo metastasis assays, including lung metastasis via tail vein injection and liver metastasis via spleen injection, as previously described (29). We transduced SK-Hep1 and Huh7 cells with the lentivirus vector carrying a dual GFP/luciferase expression system (SK-Hep1-GL and Huh7-GL cells). We then transduced SK-Hep1-GL Huh7 cells with shEV or shCLIC1 and with siRNAs targeting CLIC1 (siCLIC1) or scrambled sequences (siNC). We injected $1 \times 10^{6}$ SK-Hep1 cells in $200 \mu \mathrm{L}$ PBS per mouse into BALB/c null mice (BALB/cAnN.CgFoxn1 ${ }^{n u} /$ CrlNarl) through the tail veins or $5 \times 10^{5}$ Huh7 cells in $100 \mu \mathrm{L}$ PBS per mouse spleen for lung and liver metastasis, respectively. To compare the effect of IAA-94 treatment on tumor metastasis, mice 

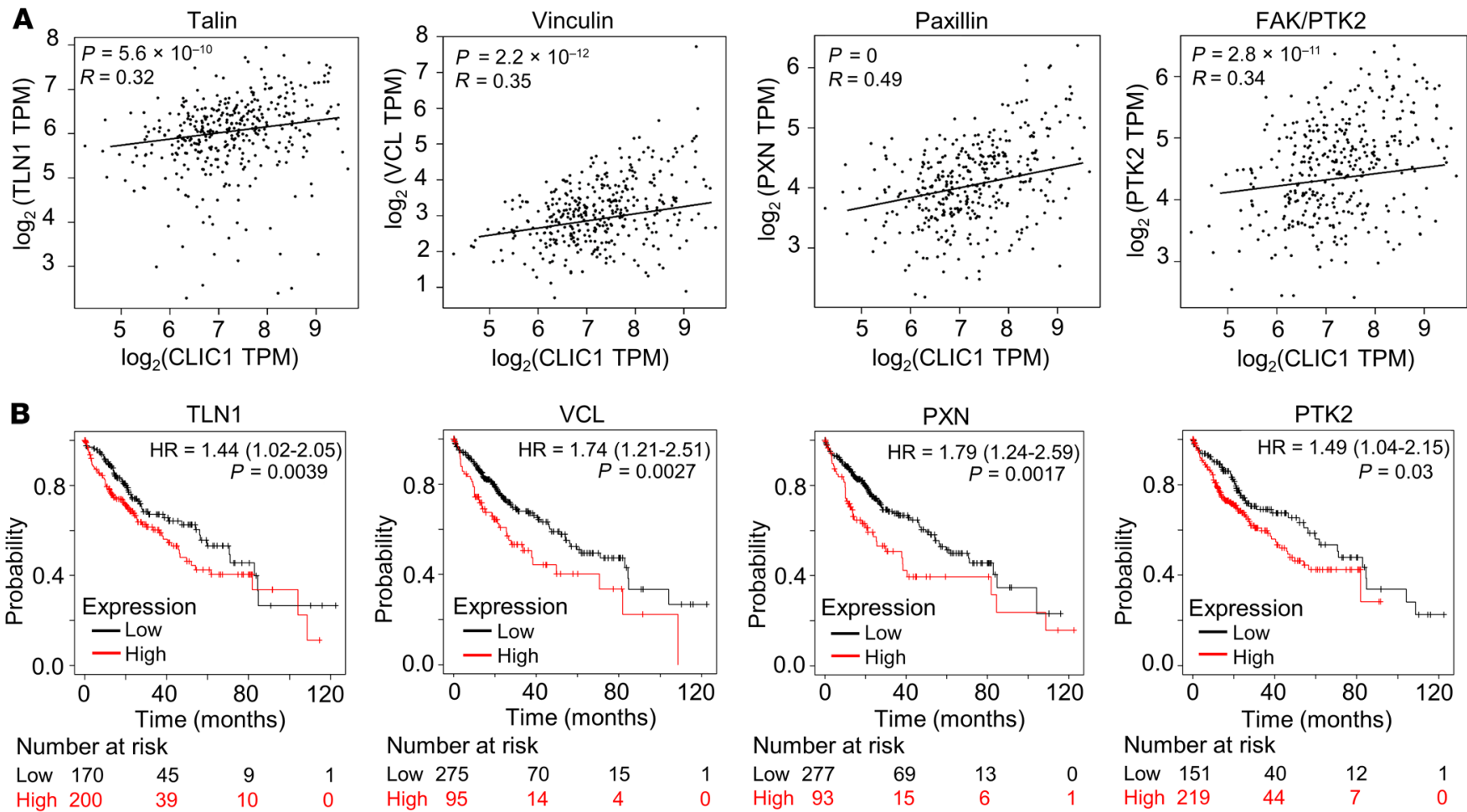

Number at risk

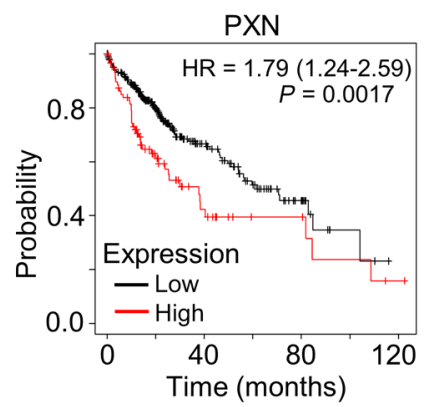

Number at risk

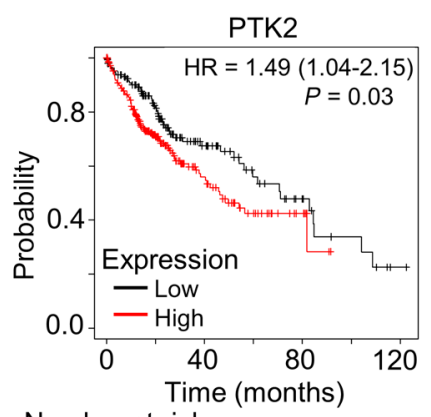

$\begin{array}{llllllll}\text { Low } 275 & 70 & 15 & 1 & \text { Low } 277 & 69 & 13 & 0 \\ \text { High } 95 & 14 & 4 & 0 & \text { High } 93 & 15 & 6 & 1\end{array}$

Number at risk

Figure 7. The expression levels of adhesion-related effectors predict poor clinical outcomes in HCC. A cohort of 370 cases of HCC was retrieved from TCGA. (A) The mRNA levels of cell-matrix adhesion markers, including talin, vinculin, paxillin, and FAK, were correlated to the CLIC1 mRNA levels by using the Pearson correlation. (B) Kaplan-Meier curves and log-rank tests for the probability of survival of 370 patients with HCC with high and low expression levels of talin (TLN1), vinculin (VCL), paxillin (PXN), and FAK (PTK2) in HCC patients. Stratification of patients into low- and high-expression subgroups was based on the optimal $P$ values, which were determined by the log-rank tests.

were intraperitoneally injected with DMSO or IAA-94 $(50 \mathrm{mg} / \mathrm{kg}$ of body weight). We used the IVIS image analysis system (Xenogen) to monitor the location and relative amounts of these transduced cells in mice at the indicated time points. Quantitative analysis of lung or liver metastasis was performed by using Living Image software (version 4.0, PerkinElmer). The lung or liver sections were taken, followed by the analysis of fluorescence-positive cells to determine xenografttumor invasion and metastasis.

Immunofluorescence staining. We conducted immunofluorescence staining to track the expression and subcellular location of the indicated proteins, as previously described (50). In brief, we first fixed cells in $4 \%$ paraformaldehyde, permeabilized the plasma membrane in $0.1 \%$ Triton X-100, blocked the background signals with a buffer containing $1 \%$ BSA, $0.1 \%$ Tween 20 for 30 minutes, treated the cells with primary antibodies (talin, vinculin, paxillin, FAK, PIP5K1A; 1:200) overnight at $4^{\circ} \mathrm{C}$, washed the samples with PBST 3 times, and then added secondary antibodies (phalloidin-Alexa488 or -Alexa594; 1:200 in blocking buffer) for 30 minutes. We counterstained the nuclei with DAPI for 5 minutes and mounted the samples with Prolong Gold antifade reagent. We inspected and analyzed the results using a confocal microscope (SP8, Leica) and Leica Application Suite Core X software (version 3.3.0).

Detection of PI $(4,5) P 2$. We determined the amount of PI $(4,5) \mathrm{P} 2$ by transfecting cells with a GFP plasmid to express PLCdelta, which contains the PI(4, 5)P2 lipid selective $\mathrm{PH}$ domain as a fluorescence translocation biosensor to determine the concentration of PI $(4,5) \mathrm{P} 2$ lipids (51). Fluorescence intensity and quantification were analyzed by ImageJ software (version 1.43).
Time-lapse imaging. We labeled the target proteins with GFP or RFP. We trypsinized and reseeded cells onto a dish coated with fibronectin (10 ng/mL). Cells with fluorescence-tagged proteins were monitored by using a Leica SP8 inverted microscope with an enclosed incubator (5\% $\left.\mathrm{CO}_{2}, 374^{\circ} \mathrm{C}\right)$. The intensity and performance of the fluorescence were analyzed by Leica Application Suit Core X software (version 3.3.0).

Wound healing assay, Transwell migration, and invasion assay. We assayed cell migration and invasion with previously published protocols (52).

Immunoprecipitation. We conducted immunoprecipitation using previously published protocols (53). In brief, SK-Hep1-shEV and SK-Hep1-shCLIC1 cells were cultured in DMEE with 10\% FBS and harvested for IP as growing up to $80 \%$ confluence. After being washed, cells were lysed in a buffer containing $50 \mathrm{mM}$ Tris ( $\mathrm{pH}$ 8.0), $150 \mathrm{mM}$ $\mathrm{NaCl}, 1 \%$ Triton $\mathrm{X}-100$, and protease and phosphatase inhibitors (Roche) for 15 minutes. Cell debris were removed by centrifugation at $13,539 \mathrm{~g}$ at $4^{\circ} \mathrm{C}$ for 15 minutes. An equal amount of protein $(1 \mathrm{mg})$ was subjected to immunoprecipitation with the corresponding primary antibodies and Protein G Mag Sepharose Xtra (Blossom Biotechnologies, Inc.) according to the manufacturer's instructions. Nonimmune IgG or Protein G-Sepharose beads were used as negative controls.

TCGA human HCC cohorts and data processing. An HCC cohort containing 370 cases (including 168, 84, 82, and 6 cases at tumor stage I, II, III, and IV, respectively) was retrieved from The Cancer Genome Atlas (TCGA) database, queried by CLIC1, HCC, survival rate, and gene expression as keywords. The expression of CLIC1 in patients was sorted and survival plots were analyzed by using the UCSC 

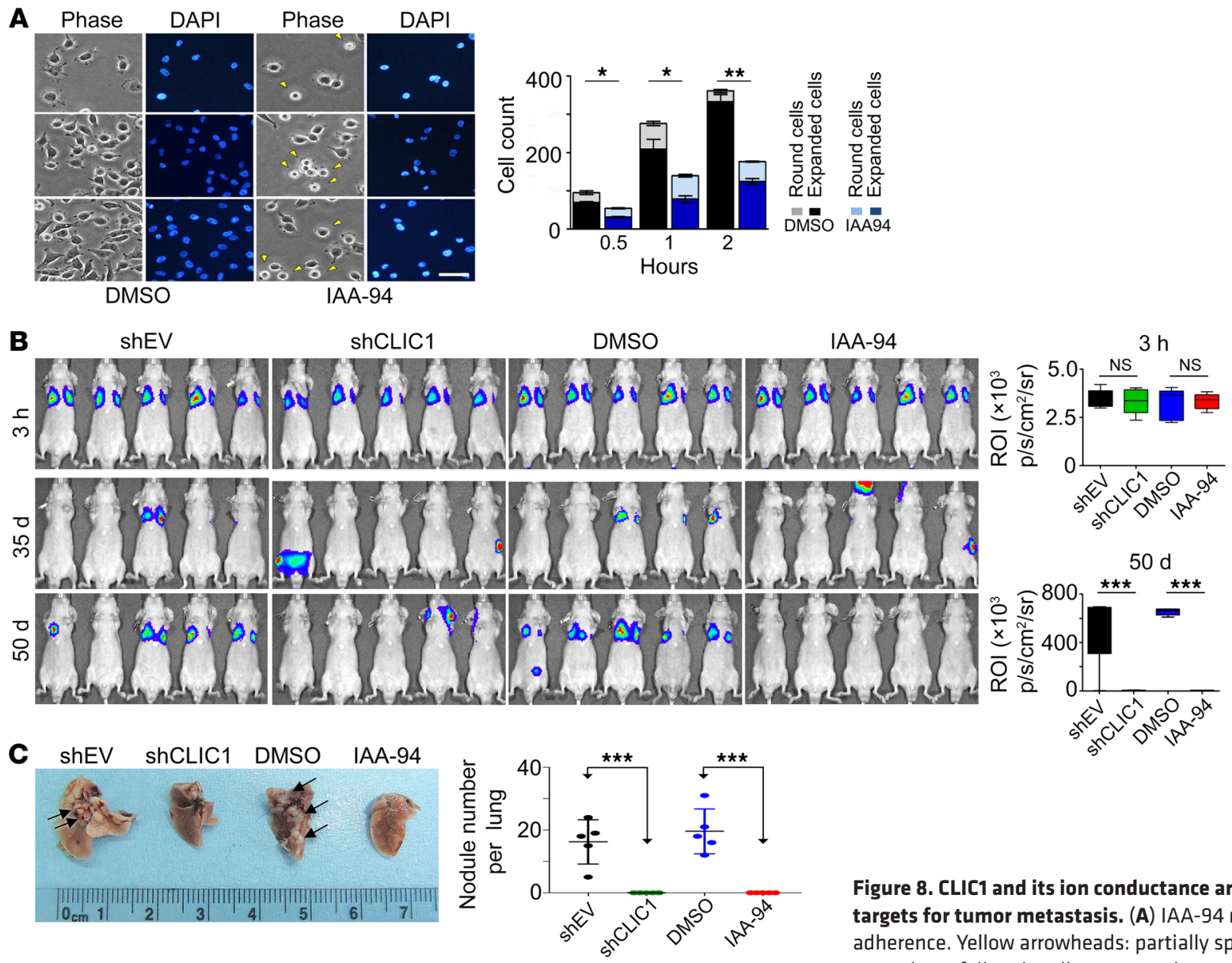

Figure 8. CLIC1 and its ion conductance are therapeutic targets for tumor metastasis. (A) IAA-94 reduced cell adherence. Yellow arrowheads: partially spread cells, representing a failure in adherence to the stratum. Scale bar: $50 \mu \mathrm{m}$. Statistical analysis was performed by using 2-tailed Student's $t$ test between 2 groups ( 4 fields/dish, 2 independent experiments; ${ }^{*} P<0.05,{ }^{* *} P<0.01$ ). (B) Xenograft lung metastasis monitored by Xenogen IVIS at different time points after injection of luciferase-transduced SK-Hep1 cells via the tail veins. Right panels: The box-and-whisker plots show the mean and interquartile ranges of the tumor-associated fluorescence intensity 3 hours (upper) or 50 days (lower) after the injection. Statistical analysis was performed by using Mann-Whitney $U$ test between 2 groups ( $n=5$ mice/group; NS, no statistical significance; ${ }^{* *} P<$ 0.001. (C) Representative lung tissues and corresponding dot plot. Arrows indicate tumor nodules. Statistical analysis was performed by using Mann-Whitney $U$ test between 2 groups ( $n=5$ mice/group). (D) Schematic of the CLIC1directed PIP5K/PIP $/$ /talin/integrin signaling pathway to initiate the assembly and signaling of nascent cell-matrix adhesions. Left: at inactive status; right: in response to chemo- or mechanotaxis, or in tumors with aberrant upregulation of CLIC1. Aberrant upregulation of CLIC1 leads to the PIP5K/PIP $/$ talin/integrin signaling to promote tumor invasion and metastasis.

Inactive status

Vin

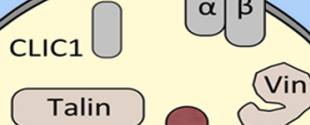
G-actin

In response to stimuli

Aberrant CLIC1 upregulation in tumors

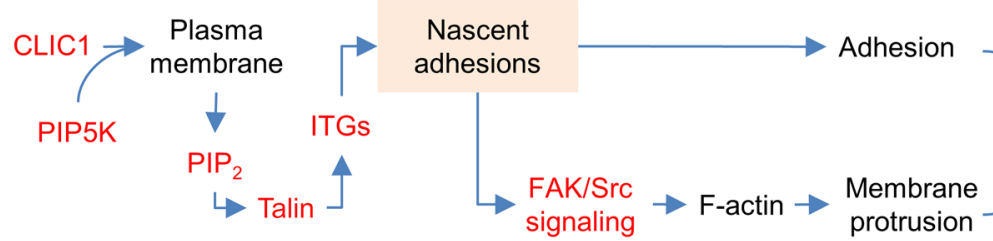

Directional

migration 
Xena Functional Genomics Explorer (https://xenabrowser.net) and SurvExpress (http://bioinformatica.mty.itesm.mx:8080/Biomatec/ SurvivaX.jsp). The cumulative survival curves for talin, vinculin, paxillin, and FAK were performed by using the software of Kaplan-Meier Plotter (https://kmplot.com/analysis/index.php?p=service) (34).

Patients, tissue arrays, immunohistochemistry. Human HCC tissue arrays containing 45 cases of chronic hepatitis B-associated and 45 cases of chronic hepatitis C-associated HCC were obtained from the Taiwan Liver Cancer Network (53). These patients underwent hepatectomy in 2008 in our hospital. The average follow-up time for these patients was 41 months (ranging from 2 to 106 months) (53). The IHC scores (calculated as the percentage of positive cells $\times$ IHC intensity [range 0-3]) were determined by using an automation system, inForm Advanced Image Analysis Software (version 2.3, PerkinElmer).

Statistics. Statistical analyses were performed using GraphPad Prism 8 (GraphPad Software). A Student's $t$ test (2-tailed), MannWhitney $U$ test, or 1-way or 2-way ANOVA with Dunnett's or Tukey's multiple comparisons test was performed. One-way ANOVA with Dunnett's multiple comparisons test was used for comparisons with the control group, 1-way ANOVA with Tukey's multiple comparisons test was used for comparisons between groups, and 2-way ANOVA with Tukey's multiple comparisons test was used for pairwise comparisons. Unless otherwise noted, all in vitro experiments were performed at least 2 independent times, and values represent the mean \pm SD. $P$ values less than 0.05 were considered significant.

Study approval. The Internal Review Board approved specimen collection procedures for HCC for the Medical Ethics of Chang Gung Memorial Hospital (201700344A3). All experimental procedures involving mice were approved by the Animal Care and Use Committee of Chang Gung
Memorial Hospital (Taiwan) and developed by the Guide for the Care and Use of Laboratory Animals (National Academies Press, 1985).

\section{Author contributions}

SYH conceived the concept, designed the studies, and secured funding. JMP and SHL performed the experiments and acquired and analyzed data. MCY collected the clinical samples and data. SYH and JMP wrote the manuscript.

\section{Acknowledgments}

We are grateful to Pien-Chien Huang at Johns Hopkins University for critical review of this manuscript. We thank Hsiao-Ling Huang for excellent assistance, Donia Alson for English review, the Microscopy Core, Center for Advanced Molecular Imaging, Laboratory Animal Center, and Clinical Proteomics Core of the Chang Gung Memorial Hospital for their technical assistance, the Taiwan Liver Cancer Network for the HCC tissue arrays, and the National RNAi Core of Taiwan for the lentivirus-based shRNA clones. This work was supported by research grants from the Chang Gung Medical Foundation (CMRPG3K0681-2, OMRPG3H0011), National Health Research Institute (NHRI-EX10610408BI, NHRI-EX109-10806BI), and the Ministry of Science and Technology (MOST103-2314-B-182A-117-MY3; MOST1072314-B-182A-020-MY3), Taiwan.

Address correspondence to: Sen-Yung Hsieh, Department of Gastroenterology and Hepatology, Chang Gung Memorial Hospital, Taoyuan 333, Taiwan. Phone: 886.975368031; Email: siming@ cgmh.org.tw or siming.shia@msa.hinet.net.
1. Yamada KM, Mayor R. Editorial overview: cell dynamics in development, tissue remodelling, and cancer. Curr Opin Cell Biol. 2016;42:iv-vi.

2. Pandya P, Orgaz JL, Sanz-Moreno V. Modes of invasion during tumour dissemination. $\mathrm{Mol}$ Oncol. 2017;11(1):5-27.

3. Humphries JD, Chastney MR, Askari JA, Humphries MJ. Signal transduction via integrin adhesion complexes. Curr Opin Cell Biol. 2019;56:14-21.

4. Lo SH, Chen LB. Focal adhesion as a signal transduction organelle. Cancer Metastasis Rev. 1994;13(1):9-24.

5. Calderwood DA, Campbell ID, Critchley DR. Talins and kindlins: partners in integrin-mediated adhesion. Nat Rev Mol Cell Biol. 2013;14(8):503-517.

6. Kim C, Ye F, Ginsberg MH. Regulation of integrin activation. Annu Rev Cell Dev Biol. 2011;27:321-345.

7. Ye F, Lagarrigue F, Ginsberg MH. SnapShot: talin and the modular nature of the integrin adhesome. Cell. 2014;156(6):1340-1340.e1.

8. Goult BT, Yan J, Schwartz MA. Talin as a mechanosensitive signaling hub. JCell Biol. 2018;217(11):3776-3784.

9. Iwamoto DV, Calderwood DA. Regulation of integrin-mediated adhesions. Curr Opin Cell Biol. 2015;36:41-47.

10. Warner H, Wilson BJ, Caswell PT. Control of adhesion and protrusion in cell migration by Rho GTPases. Curr Opin Cell Biol. 2019;56:64-70.

11. Lo SH. C-terminal tensin-like (CTEN): a promis- ing biomarker and target for cancer. Int J Biochem Cell Biol. 2014;51:150-154.

12. Strilic B, Offermanns S. Intravascular survival and extravasation of tumor cells. Cancer Cell. 2017;32(3):282-293.

13. Singh H, Cousin MA, Ashley RH. Functional reconstitution of mammalian 'chloride intracellular channels' CLIC1, CLIC4 and CLIC5 reveals differential regulation by cytoskeletal actin. FEBS J. 2007;274(24):6306-6316.

14. Goodchild SC, et al. Oxidation promotes insertion of the CLIC1 chloride intracellular channel into the membrane. Eur Biophys J. 2009;39(1):129-138.

15. Ulmasov B, et al. CLIC1 null mice demonstrate a role for CLIC1 in macrophage superoxide production and tissue injury. Physiol Rep. 2017;5(5):e13169.

16. Ulmasov B, Bruno J, Woost PG, Edwards JC. Tissue and subcellular distribution of CLIC1. BMC Cell Biol. 2007;8:8.

17. Hare JE, Goodchild SC, Breit SN, Curmi PM, Brown LJ. Interaction of human chloride intracellular channel protein 1 (CLIC1) with lipid bilayers: a fluorescence study. Biochemistry. 2016;55(27):3825-3833.

18. Singh H, Ashley RH. Redox regulation of CLIC1 by cysteine residues associated with the putative channel pore. Biophys J. 2006;90(5):1628-1638.

19. Peter B, Fanucchi S, Dirr HW. A conserved cationic motif enhances membrane binding and insertion of the chloride intracellular channel protein 1 transmembrane domain. Eur Biophys J. 2014;43(8-9):405-414.

20. Liu B, et al. Chloride intracellular channel 1 (CLIC1) contributes to modulation of cyclic AMP-activated whole-cell chloride currents in human bronchial epithelial cells. Physiol Rep. 2018;6(2):e13508.

21. Jia N, Dong S, Zhao G, Gao H, Li X, Zhang H. CLIC1 overexpression is associated with poor prognosis in pancreatic ductal adenocarcinomas. J Cancer Res Ther. 2016;12(2):892-896.

22. Zhang S, et al. Chloride intracellular channel 1 is overexpression in hepatic tumor and correlates with a poor prognosis. APMIS. 2013;121(11):1047-1053.

23. Wang JW, et al. Identification of metastasis-associated proteins involved in gallbladder carcinoma metastasis by proteomic analysis and functional exploration of chloride intracellular channel 1. Cancer Lett. 2009;281(1):71-81.

24. Petrova DT, et al. Expression of chloride intracellular channel protein 1 (CLIC1) and tumor protein D52 (TPD52) as potential biomarkers for colorectal cancer. Clin Biochem. 2008;41(14-15):1224-1236.

25. Chen CD, et al. Overexpression of CLIC1 in human gastric carcinoma and its clinicopathological significance. Proteomics. 2007;7(1):155-167.

26. Chang YH, Wu CC, Chang KP, Yu JS, Chang YC, Liao PC. Cell secretome analysis using hollow 
fiber culture system leads to the discovery of CLIC1 protein as a novel plasma marker for nasopharyngeal carcinoma. J Proteome Res. 2009;8(12):5465-5474.

27. Ding $\mathrm{Q}$, et al. CLIC1 overexpression is associated with poor prognosis in gallbladder cancer. Tumour Biol. 2015;36(1):193-198.

28. Li RK, Zhang J, Zhang YH, Li ML, Wang M, Tang JW. Chloride intracellular channel 1 is an important factor in the lymphatic metastasis of hepatocarcinoma. Biomed Pharmacother. 2012;66(3):167-172.

29. Peng JM, et al. Actin cytoskeleton remodeling drives epithelial-mesenchymal transition for hepatoma invasion and metastasis in mice. Hepatology. 2018;67(6):2226-2243.

30. Gómez-Cuadrado L, Tracey N, Ma R, Qian B, Brunton VG. Mouse models of metastasis: progress and prospects. Dis Model Mech. 2017;10(9):1061-1074.

31. Kadry YA, Calderwood DA. Chapter 22: structural and signaling functions of integrins. Biochim Biophys Acta Biomembr. 2020;1862(5):183206.

32. De Craene JO, Bertazzi DL, Bär S, Friant S. Phosphoinositides, major actors in membrane trafficking and lipid signaling pathways. Int J Mol Sci. 2017;18(3):E634.

33. Hammond GR. Does PtdIns(4,5)P2 concentrate so it can multi-task? Biochem Soc Trans. 2016;44(1):228-233.

34. Menyhárt O, Nagy Á, Győrffy B. Determining consistent prognostic biomarkers of overall survival and vascular invasion in hepatocellular carcinoma. R Soc Open Sci. 2018;5(12):181006.

35. Edwards JC. The CLIC1 chloride channel is regulated by the cystic fibrosis transmembrane con- ductance regulator when expressed in Xenopus oocytes. J Membr Biol. 2006;213(1):39-46.

36. Skaper SD, Facci L, Giusti P. Intracellular ion channel CLIC1: involvement in microgliamediated $\beta$-amyloid peptide(1-42) neurotoxicity. Neurochem Res. 2013;38(9):1801-1808.

37. Orłowski A, et al. PIP2 and talin join forces to activate integrin. J Phys Chem B. 2015;119(38):12381-12389.

38. Wang JH. Pull and push: talin activation for integrin signaling. Cell Res. 2012;22(11):1512-1514.

39. Salao K, et al. CLIC1 regulates dendritic cell antigen processing and presentation by modulating phagosome acidification and proteolysis. Biol Open. 2016;5(5):620-630.

40. Ye X, McLean MA, Sligar SG. Conformational equilibrium of talin is regulated by anionic lipids. Biochim Biophys Acta. 2016;1858(8):1833-1840.

41. Argenzio E, et al. Profilin binding couples chloride intracellular channel protein CLIC4 to RhoA-mDia2 signaling and filopodium formation. J Biol Chem. 2018;293(50):19161-19176.

42. Argenzio E, et al. CLIC4 regulates cell adhesion and $\beta 1$ integrin trafficking. JCell Sci. 2014;127(pt 24):5189-5203.

43. Dozynkiewicz MA, et al. Rab25 and CLIC3 collaborate to promote integrin recycling from late endosomes/lysosomes and drive cancer progression. Dev Cell. 2012;22(1):131-145.

44. Macpherson IR, et al. CLIC3 controls recycling of late endosomal MT1-MMP and dictates invasion and metastasis in breast cancer. J Cell Sci. 2014;127(pt 18):3893-3901.

45. Barbieri F, et al. Repurposed biguanide drugs in glioblastoma exert antiproliferative effects via the inhibition of intracellular chloride channel 1
Activity. Front Oncol. 2019;9:135.

46. Gurski LA, Knowles LM, Basse PH, Maranchie JK, Watkins SC, Pilch J. Relocation of CLIC1 promotes tumor cell invasion and colonization of fibrin. Mol Cancer Res. 2015;13(2):273-280.

47. Setti M, et al. Functional role of CLIC1 ion channel in glioblastoma-derived stem/progenitor cells. J Natl Cancer Inst. 2013;105(21):1644-1655.

48. Barbieri F, et al. Inhibition of chloride intracellular channel 1 (CLIC1) as biguanide class-effect to impair human glioblastoma stem cell viability. Front Pharmacol. 2018;9:899.

49. Hsieh SY, Zhuang FH, Wu YT, Chen JK, Lee YL. Profiling the proteome dynamics during the cell cycle of human hepatoma cells. Proteomics. 2008;8(14):2872-2884.

50. Lo SJ, et al. A novel interaction of nucleophosmin with BCL2-associated X protein regulating death evasion and drug sensitivity in human hepatoma cells. Hepatology. 2013;57(5):1893-1905.

51. Gulshan K, et al. PI(4,5)P2 is translocated by $\mathrm{ABCA1}$ to the cell surface where it mediates apolipoprotein A1 binding and nascent HDL assembly. Circ Res. 2016;119(7):827-838.

52. Peng JM, et al. Actin cytoskeleton remodeling drives epithelial-mesenchymal transition for hepatoma invasion metastasis. Hepatology. 2018;67(6):2226-2243.

53. Bera R, et al. Functional genomics identified a novel protein tyrosine phosphatase receptor type F-mediated growth inhibition in hepatocarcinogenesis. Hepatology. 2014;59(6):2238-2250.

54. Al-Momany A, Li L, Alexander RT, Ballermann BJ. Clustered PI $(4,5) \mathrm{P}_{2}$ accumulation and ezrin phosphorylation in response to CLIC5A. JCell Sci. 2014;127(Pt 24):5164-5178. 